\title{
SYMMETRY OF THE DEFINITION OF DEGENERATION IN TRIANGULATED CATEGORIES
}

\author{
MANUEL SAORÍN AND ALEXANDER ZIMMERMANN
}

\begin{abstract}
Module structures of an algebra on a fixed finite dimensional vector space form an algebraic variety. Isomorphism classes correspond to orbits of the action of an algebraic group on this variety and a module is a degeneration of another if it belongs to the Zariski closure of the orbit. Riedtmann and Zwara gave an algebraic characterisation of this concept in terms of the existence of short exact sequences. Jensen, Su and Zimmermann, as well as independently Yoshino, studied the natural generalisation of the Riedtmann-Zwara degeneration to triangulated categories. The definition has an intrinsic non-symmetry. Suppose that we have a triangulated category in which idempotents split and either for which the endomorphism rings of all objects are artinian, or which is the category of compact objects in an algebraic compactly generated triangulated $K$-category. Then we show that the non-symmetry in the algebraic definition of the degeneration is inessential in the sense that the two possible choices which can be made in the definition lead to the same concept.
\end{abstract}

\section{INTRODUCTION}

For a finite dimensional $K$-algebra over an algebraically closed field $K$ the set of $d$ dimensional $A$-modules is just the space of $K$-algebra homomorphisms from $A$ to the algebra of $d$ by $d$ matrices over $K$. It carries therefore the structure of an algebraic variety $\bmod (A, d)$, and allows a $G L_{d}(K)$-action given by conjugation of matrices. $G L_{d}(K)$-orbits correspond to isomorphism classes of modules, and we say that a $d$-dimensional module $M$ corresponding to the point $m \in \bmod (A, d)$ degenerates to the module $N$ with corresponding point $n \in$ $\bmod (A, d)$ if $n$ belongs to the Zariski-closure of the orbit $G L_{d}(K) \cdot m$. We write in this case $M \leq_{\operatorname{deg}} N$. It is clear that $\leq_{\operatorname{deg}}$ is a partial order on the set of isomorphism classes of finite dimensional $A$-modules. Zwara and Riedtmann defined another relation between $A$-modules, namely $M \leq_{\text {Zwara }} N$ if and only if there is a finite dimensional $A$-module $Z$ and a short exact sequence $0 \rightarrow N \rightarrow M \oplus Z \rightarrow Z \rightarrow 0$. Moreover, they showed in [9, 20]

$$
M \leq_{\operatorname{deg}} N \Leftrightarrow M \leq_{\text {Zwara }} N .
$$

Zwara showed in [19] by a purely algebraic arguments that in the category of finite dimensional modules over an algebra there is $Z$ and a short exact sequence $0 \rightarrow N \rightarrow M \oplus Z \rightarrow$ $Z \rightarrow 0$ if and only if there is $Z^{\prime}$ and a short exact sequence $0 \rightarrow Z^{\prime} \rightarrow M \oplus Z^{\prime} \rightarrow N \rightarrow 0$.

In joint work with Jensen and $\mathrm{Su}$ [4, and independently by Yoshino in [15] for the (triangulated) stable category of maximal Cohen Macaulay modules over local Gorenstein $k$-algebras, the concept $\leq_{\text {Zwara }}$ was generalised in the obvious way to general triangulated categories. More precisely, for a triangulated category $\mathcal{T}$ we define for two objects $M$ and $N$ that $M \leq_{\Delta} N$ if and only if there is an object $Z$ and a distinguished triangle $Z \stackrel{\left(\begin{array}{l}u \\ v\end{array}\right)}{\longrightarrow} M \oplus Z \longrightarrow N \rightarrow Z[1]$. Yoshino insisted in the point that one should ask that the induced endomorphism $v$ of $Z$ is nilpotent. Using Fitting's lemma and possibly replacing $Z$

Date: December 16, 2016.

2010 Mathematics Subject Classification. Primary: 18E30, Secondary: 16E45, 14B05.

Key words and phrases. degeneration; triangulated category; differential graded algebra; differential graded category.

The first named author is backed by research projects from the Ministerio de Economía y Competitividad of Spain (MTM201346837-P) and the Fundación 'Séneca' of Murcia (19880/GERM/15), both with a part of FEDER funds. He thanks these institutions for their support. 
by a suitable direct summand, this is automatic if one assumes Krull-Schmidt properties and artinian endomorphism rings for all objects. We denote $M \leq_{\Delta+\text { nil }} N$ if $M \leq_{\Delta} N$ and the induced endomorphism $v$ on $Z$ is nilpotent. The concept $\leq_{\Delta}$ was used in an essential way in work of Keller and Scherotzke on Nakajima quiver varieties. [4] concentrated on partial order properties of $\leq_{\Delta}$. Further conditions guaranteeing partial order properties of $\leq_{\Delta}$ can be found for various situations in [17, [16] and [11]. In this latter reference a geometric setting was developed replacing the module variety $\bmod (A, d)$ for general triangulated categories, mimicking for this purpose Yoshino's scheme theoretic approach [15]. Various results were given that ensure that $\leq_{\Delta}$ or $\leq_{\Delta+\text { nil }}$ define partial orders on the isomorphism classes of objects of $\mathcal{T}$.

Some authors define $\leq_{\Delta}$ (resp. $\left.\leq_{\Delta+\text { nil }}\right)$ by the existence of a distinguished triangle

$$
N \rightarrow M \oplus Z \stackrel{\left(u_{r} v_{r}\right)}{\longrightarrow} Z \rightarrow N[1]
$$

and some define it as the existence of a distinguished triangle

$$
Z \stackrel{\left(\begin{array}{l}
u_{\ell} \\
v_{\ell}
\end{array}\right)}{\longrightarrow} M \oplus Z \rightarrow N \rightarrow Z[1]
$$

Passing to the opposite category the two definitions relate to each other. Note that the opposite category of a triangulated category is triangulated as well. However, we show in this paper that actually the situation is even better. The two possible definitions lead to the same relation on the isomorphism classes of objects in two important cases. Our main result is the following. In its statement and in the rest of the paper 'artinian ring' means 'left and right artinian'.

Theorem 1. Let $K$ be a commutative ring and let $\mathcal{T}$ be a $K$-linear triangulated category satisfies one of the following two hypotheses

(a) Idempotents split in $\mathcal{T}$ and all endomorphism algebras of objects are artinian,

(b) $\mathcal{T}$ is the category of compact objects in an algebraic compactly generated triangulated $K$-category.

Then for any objects $M, N$ of $\mathcal{T}$, the following assertions are equivalent:

(1) There is an object $Z_{\ell}$ of $\mathcal{T}$ and a distinguished triangle in $\mathcal{T}$

$$
Z_{\ell} \stackrel{\left(\begin{array}{l}
v_{\ell} \\
u_{\ell}
\end{array}\right)}{\longrightarrow} Z_{\ell} \oplus M \longrightarrow N \longrightarrow Z_{\ell}[1],
$$

where $v_{\ell}$ is a nilpotent endomorphism of $Z_{\ell}$.

(2) There is an object $Z_{r}$ of $\mathcal{T}$ and a distinguished triangle in $\mathcal{T}$

$$
N \longrightarrow M \oplus Z_{r} \stackrel{\left(u_{r} \quad v_{r}\right)}{\longrightarrow} Z_{r} \longrightarrow N[1]
$$

where $v_{r}$ is a nilpotent endomorphism of $Z_{r}$.

It should be noted that since any (left or right) artinian ring is semiperfect (see [12, Examples VIII.4])), under the first situation of the theorem, the category $\mathcal{T}$ is Krull-Schmidt (see [2, Theorem A.1]). Moreover, under this hypothesis the assumption that $v_{\ell}$ (resp. $v_{r}$ ) is nilpotent is inessential. Indeed, a Fitting lemma type argument can then by applied and this shows that we can split off a trivial distinguished triangle as direct factor such that the remaining direct factor distinguished triangle satisfies the nilpotency hypothesis (cf Remark 12 below for more details). We cannot avoid the artinian hypothesis in the first and the nilpotency hypothesis in both cases. The proof in the first case follows Zwara's arguments in [19] in the classical case, but there are quite a few subtleties arising by the nonuniqueness in the TR3-axiom of triangulated categories. Zwara frequently uses pushouts and pullbacks and in particular universal properties which come along with these concepts. We replace these constructions by homotopy cartesian squares, and have to cope with the lack of uniqueness of the related construction. The proof in the second case is much more 
involved and heavily uses the concepts developed in [10]. The main idea in this approach is to use a dualisation functor like the $K$-duals for ordinary $K$-algebras $A$. However the situation is more involved here. The hypothesis that the triangulated category is the category of compact objects in an algebraic and compactly generated triangulated category gives that it is actually equivalent to the category of compact objects in the derived category of some small dg-category. Then, the new approach is to see this derived category as the derived category $\mathcal{D}(A)$ of some dg algebra without unit $A$, but with sufficiently many idempotents in a certain sense. Then, it can be shown that one may dualise with respect to $A$, using the derived functor of the suitable contravariant Hom functor to $A$. Further we use in particular the main result of [11] in full generality. The theory of dg algebras with enough idempotents parallels in a certain sense the development of dg categories as given by Keller but the situation is new. The approach is presented in [10], and we believe that such a theory is highly useful and should provide many further applications.

The paper is organised as follows. In Section 1 we give a summary of the contents of reference [10], in order to provide the vocabulary needed to understand the proof of the main result in the main body of the paper, without being obliged to go into the full details of that reference. In Section 2 we give the relevant background, facts and definitions of degenerations of objects in module categories, as well as in triangulated categories as it was shown in our earlier papers [4, 11. Section 3 then proves the main result Theorem 1 under the hypothesis (a), i.e. in case all objects in the triangulated category have artinian endomorphism ring. The final Section 4 then gives the proof of Theorem 1 under hypothesis (b), i.e. in the case of a triangulated category which is the category of compact objects in an algebraic compactly generated triangulated category.

\section{Review ON tRiangulated CATEGORIES, DG-CATEGORIES AND DG-ALGEBRAS With ENOUGH IDEMPOTENTS}

For the proof of Theorem 1 under hypothesis (b), which will cover Section 4, we shall need some concepts and statements from the theory of dg-algebras, dg-categories and triangulated categories in general which are not standard. In particular in case of categories which do not satisfy Krull-Schmidt theorem, we proceed by considering dg algebras without units, but having enough idempotents. The complete theory can be found in [10]. In order to facilitate the reading we summarize the results of this latter reference and introduce this way also the notations used in Section 4. All throughout the rest of the paper, let $K$ be a commutative ring with unit and all categories which appear all assumed to be $K$-categories. The unadorned symbol $\otimes$ will stand for the tensor product over $K$.

1.1. $\mathbf{d g}$ categories and $\mathbf{d g}$ functors. Recall that a differential graded ( $d g) K$-module is a $\mathbb{Z}$-graded $K$-module $V$ with a graded endomorphism $d: V \longrightarrow V$ of degree 1 and square 0 , called the differential (here and all throughout the paper, when the term 'differential' is used to denote a graded map $d$, it will be assumed, without further remark, that $d \circ d=0$ and that $d$ is graded and of degree +1$)$. We denote by $D g-K$ or $\mathcal{C}_{d g} K$ the category of $\operatorname{dg} K$-modules. The morphism space $\operatorname{HOM}_{K}(V, W)$ in this category is again a dg $K$-module, where the homogeneous component of degree $n$, denoted $\operatorname{HOM}_{K}^{n}(V, W)$, consists of the homogeneous morphisms of degree $n$. The differential is given by $d_{H o m}(\alpha)=d_{W} \circ \alpha-(-1)^{|\alpha|} \alpha \circ d_{V}$, for any homogeneous morphism $\alpha \in \operatorname{HOM}_{K}(V, W)$, where $|\cdot|$ denotes the degree.

A $d g$ category $\mathcal{A}$ (see [5] or [6]) is a category such that the morphism spaces are $\mathrm{dg} K$ modules and the composition map $\operatorname{Hom}_{\mathcal{A}}(B, C) \otimes \operatorname{Hom}_{\mathcal{A}}(A, B) \longrightarrow \operatorname{Hom}_{\mathcal{A}}(A, C)$ satisfies Leibniz rule $d(g \circ f)=d(g) \circ f+(-1)^{|g|} g \circ d(f)$, for all homogeneous morphisms $f \in$ $\operatorname{Hom}_{\mathcal{A}}(A, B)$ and $g \in \operatorname{Hom}_{\mathcal{A}}(B, C)$, where, abusing notation, we have denoted by $d$ the differential on any of the appearing $H o m$ spaces. The category $D g-K$ (denoted by $\mathcal{C}_{d g} K$ in [6]) is the prototype of a dg category. With any such category, one canonically associates its 0 -cycle category $Z^{0} \mathcal{A}$ and its 0-homology category $\mathcal{H}^{0} \mathcal{A}$. Both of them have the same objects as $\mathcal{A}$, and as morphisms one puts $\operatorname{Hom}_{Z^{0} \mathcal{A}}\left(A, A^{\prime}\right)=Z^{0}\left(\mathcal{A}\left(A, A^{\prime}\right)\right)$ and $\operatorname{Hom}_{H^{0} \mathcal{A}}\left(A, A^{\prime}\right)=$ 
$H^{0}\left(\mathcal{A}\left(A, A^{\prime}\right)\right)$, for all $A, A^{\prime} \in \mathrm{Ob}(\mathcal{A})$, the composition of morphisms in both cases being induced by the composition in $\mathcal{A}$. A dg functor $F: \mathcal{A} \longrightarrow \mathcal{B}$ between dg categories is just a functor which preserves the grading and the differential of Hom spaces. Any dg functor $F: \mathcal{A} \longrightarrow \mathcal{B}$ induces corresponding functors $F=Z^{0} F: Z^{0} \mathcal{A} \longrightarrow Z^{0} \mathcal{B}$ and $F: H^{0} F$ : $H^{0} \mathcal{A} \longrightarrow H^{0} \mathcal{B}$.

Associated to $\mathcal{A}$, there is also the opposite $d g$ category $\mathcal{A}^{o p}$ and, given another $\operatorname{dg}$ category $\mathcal{B}$, there is a definition of tensor product of $d g$ categories $\mathcal{A} \otimes \mathcal{B}$. A homological natural transformation of dg functors $\tau: F \longrightarrow G$ is a natural transformation such that $\tau_{A} \in Z^{0}\left(\operatorname{Hom}_{\mathcal{B}}(F(A), G(A))\right)$, for any object $A \in \mathcal{A}$. If we have dg functors $F: \mathcal{A} \longrightarrow \mathcal{B}$ and $G: \mathcal{B} \longrightarrow \mathcal{A}$, then we have induced dg functors $\mathcal{A}^{\text {op }} \otimes \mathcal{B} \longrightarrow D g-K$, given by $\operatorname{Hom}_{\mathcal{B}}(F(?), ?)$ and $\operatorname{Hom}_{\mathcal{A}}(?, G(?))$. A dg adjunction is just an adjunction $(F, G)$ of dg functors such that the natural isomorphism $\operatorname{Hom}_{\mathcal{B}}(F(?), ?) \stackrel{\cong}{\longrightarrow} \operatorname{Hom}_{\mathcal{A}}(?, G(?))$ is a homological natural transformation. See [5] and [10, Section 1] for the details concerning dg categories and $\mathrm{dg}$ functors.

1.2. dg categories and dg algebras with enough idempotents. Any small $K$-category can be viewed as an algebra with enough idempotents. The latter is a $K$-algebra $A$ with a distinguished family $\left(e_{i}\right)_{i \in I}$ of orthogonal idempotents such that $\bigoplus_{i \in I} e_{i} A=A=\bigoplus A e_{i}$. When such an algebra comes with a grading (as an algebra) such that the $e_{i}$ are homogeneous of zero degree, and with a differential $d: A \longrightarrow A$ such that $d\left(e_{i}\right)=0$, for all $i \in I$, and $d$ satisfies Leibniz rule, then $A$ or the pair $(A, d)$ is called a differential graded ( $d g)$ algebra with enough idempotents. It is also shown in [10] that such an algebra may be viewed as a small dg category with $I$ as set of objects. To any such algebra $A$ one canonically associates a (non-small) dg category $D g-A$, whose objects are right $d g A$-modules. A right $\operatorname{dg} A$ module is just a graded right $A$-module $M$ together with a differential $d_{M}: M \longrightarrow M$ such that $d_{M}(x a)=d_{M}(x) a+(-1)^{|x|} x d_{A}(a)$, for all homogeneous elements $x \in M$ and $a \in A$. Here and in the rest of the paper, unless otherwise specified, all modules are assumed to be unitary. That is, we assume that $M=M A$ in our case. We denote by $G r-A$ the category with objects the graded right $A$-modules and morphisms the graded $A$-homomorphisms of degree zero. This category comes with a canonical equivalence ?[1] :

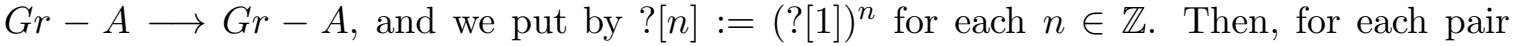
$(M, N)$ of right $\operatorname{dg} A$-modules, the corresponding space of morphisms in $D g-A$ is given by $\operatorname{HOM}_{A}(M, N)=\bigoplus_{n \in \mathbb{Z}} \operatorname{HOM}_{A}^{n}(M, N)$, where $\operatorname{HOM}_{A}^{n}(M, N):=\operatorname{Hom}_{G r-A}(M, N[n])$. The differential of $\operatorname{HOM}_{A}(M, N)$ is the restriction of the differential of $\operatorname{HOM}_{K}(M, N)$ (see the first paragraph of Section 1.1.) One similarly defines the opposite dg algebra with enough idempotents $A^{o p}$ and the tensor product $A \otimes B$ of dg algebras with enough idempotents. One then defines the dg category $A-D g$ of left $\operatorname{dg}$ modules and that of $\operatorname{dg} A-B$-bimodules, which are equivalent to $D g-A^{o p}$ and $D g-\left(B \otimes A^{o p}\right)$, respectively. This allows to treat the theories of left $\mathrm{dg}$ modules or $\mathrm{dg}$ bimodules over $\mathrm{dg}$ algebras with enough idempotents just as right dg modules.

1.3. Stable and derived category of a dg algebra with enough idempotents. The 0 cycle (resp. 0-homology) category of $D g-A$ is denoted by $\mathcal{C}(A)$ (resp. $\mathcal{H}(A)$ ). The category $\mathcal{C}(A)$ is a bicomplete abelian category, with exact sequences as in $G r-A$, and, apart from this abelian structure, it also has a Quillen exact structure, called the semi-split exact structure, where the conflations (=admissible short exact sequences) are those exact sequences which split in $G r-A$ (see [1] for the terminology and main properties of exact categories). With this latter structure $\mathcal{C}(A)$ is Frobenius, that is, $\mathcal{C}(A)$ has enough projectives and injectives and the injective objects coincide with the projective ones. The stable category of $\mathcal{C}(A)$, which is then triangulated (see [3]), is precisely $\mathcal{H}(A)$. This latter (triangulated) category is called the homotopy category of $A$. The class of quasi-isomorphisms in $\mathcal{H}(A)$ (i.e. those morphisms which induce isomorphisms on homology) is a multiplicative system compatible with the triangulation in the terminology of Verdier (see [13]). The localization of $\mathcal{H}(A)$ 
with respect to the class of quasi-isomorphism, denoted $\mathcal{D}(A)$, is the derived category of $A$. It then has a unique structure of triangulated category such that the canonical functor $q: \mathcal{H}(A) \longrightarrow \mathcal{D}(A)$ is triangulated. In [10] (see, Theorem 3.1 in that reference) it is proved that the theory of dg modules over dg algebras with enough idempotents and their homotopy and derived categories is equivalent to the corresponding theory over small $\mathrm{dg}$ categories (see [5] and [6] for the details of this latter theory). As a consequence of Keller's famous theorem (see [5, Theorem 4.3]), one gets that any algebraic compactly generated triangulated category is equivalent to $\mathcal{D}(A)$, for some dg algebra with enough idempotents $A$ (see [10, Corollary 6.10]). Recall that a triangulated category $\mathcal{T}$ is algebraic when it is equivalent to the stable category of some Frobenius exact category, and that it is called compactly generated when $\mathcal{T}$ has coproducts and there is a set of compact objects $\mathcal{C}$ in $\mathcal{T}$ such that $\bigcap_{C \in \mathcal{C}, n \in \mathbb{Z}} \operatorname{Ker}\left(\operatorname{Hom}_{\mathcal{T}}(C[n], ?)\right)=0$. Recall that an object $C$ is compact when the functor $\operatorname{Hom}_{\mathcal{T}}(C, ?): \mathcal{T} \longrightarrow A b$ preserves arbitrary coproducts.

1.4. Derived functors. A (right) dg A-module $P$ (resp. $I$ ) is homotopically projective (resp. homotopically injective) when the functor $\operatorname{HOM}_{A}(P, ?): D g-A \longrightarrow D g-K$ (resp. $\left.\operatorname{HOM}_{A}(?, I):(D g-A)^{o p} \longrightarrow D g-K\right)$ preserves acyclic dg modules, something which is equivalent to saying that the induced functor $\operatorname{Hom}_{\mathcal{H}(A)}(P$, ?) $: \mathcal{H}(A) \longrightarrow \operatorname{Mod}-K$ (resp. $\left.\operatorname{Hom}_{\mathcal{H}(A)}(?, I): \mathcal{H}(A)^{o p} \longrightarrow \operatorname{Mod}-K\right)$ vanishes on acyclic dg $A$-modules. As in the case of small dg categories, the canonical functor $q_{A}: \mathcal{H}(A) \longrightarrow \mathcal{D}(A)$ has a left adjoint functor $\Pi_{A}: \mathcal{D}(A) \longrightarrow \mathcal{H}(A)$, called the homotopically projective resolution functor, and a right adjoint $\Upsilon_{A}: \mathcal{D}(A) \longrightarrow \mathcal{H}(A)$, called the homotopically injective resolution functor, both of which are fully faithful and triangulated. They are so named because $\operatorname{Im}\left(\Pi_{A}\right.$ ) (resp. $\operatorname{Im}\left(\Upsilon_{A}\right)$ ) consists of homotopically projective (resp. homotopically injective) $\operatorname{dg} A$-modules. The counit $\pi: \Pi_{A} \circ q_{A} \longrightarrow 1_{\mathcal{H}(A)}$ (resp. unit $\iota: 1_{\mathcal{H}(A)} \longrightarrow q_{A} \circ \Upsilon_{A}$ ) of the adjunction $\left(\Pi_{A}, q_{A}\right)$ (resp. $\left(q_{A}, \Upsilon_{A}\right)$ ) has the property that $\pi_{M}$ (resp. $\left.\iota_{M}\right)$ is a quasi-isomorphism, for each $\mathrm{dg}$ module $M$, and it is even an isomorphism when $M$ is homotopically projective (resp. homotopically injective). Given a dg functor $F: D g-A \longrightarrow D g-B$ which preserves contractible dg modules, one defines its left derived functor (resp. right derived functor) $\mathbb{L} F: \mathcal{D}(A) \longrightarrow \mathcal{D}(B)$ (resp. $\mathbb{R} F: \mathcal{D}(A) \longrightarrow \mathcal{D}(B)$ ), as the composition

$$
\mathcal{D}(A) \stackrel{\Pi_{A}}{\longrightarrow} \mathcal{H}(A) \stackrel{F}{\longrightarrow} \mathcal{H}(B) \stackrel{q_{B}}{\longrightarrow} \mathcal{D}(B)
$$

(resp. $\mathcal{D}(A) \stackrel{\Upsilon_{A}}{\longrightarrow} \mathcal{H}(A) \stackrel{F}{\longrightarrow} \mathcal{H}(B) \stackrel{q_{B}}{\longrightarrow} \mathcal{D}(B)$ ). When the dg functor is contravariant, meaning that $F:(D g-A)^{o p} \longrightarrow D g-B$ a dg functor, which preserves contractibility, then we define its right derived functor $\mathbb{R} F$ as the composition

$$
\mathcal{D}(A)^{o p} \stackrel{\Pi_{A}^{o}}{\longrightarrow} \mathcal{H}(A)^{o p} \stackrel{F}{\longrightarrow} \mathcal{H}(B) \stackrel{q_{B}}{\longrightarrow} \mathcal{D}(B) .
$$

All these derived functors are triangulated since they are composition of triangulated functors. If moreover $G: D g-A \longrightarrow D g-B$ is another dg functor as above and $\tau: F \longrightarrow G$ is a homological natural transformation of $\mathrm{dg}$ functors, then one obtains corresponding natural transformations of triangulated functors, still denoted the same, $\tau: \mathbb{L} F \longrightarrow \mathbb{L} G$ and $\tau: \mathbb{R} F \longrightarrow \mathbb{R} G$ in the covariant case, and just $\tau: \mathbb{R} F \longrightarrow \mathbb{R} G$ in the contravariant case. Not only that, but any dg adjunction $(F, G)$ of dg functors gives rise to a corresponding triangulated adjunction $(\mathbb{L} F, \mathbb{R} G)$ in the covariant case, and $\left((\mathbb{R} F)^{o}, \mathbb{R} G\right)$ in the contravariant case (see [10, Proposition 7.13]).

This somehow classical picture is extended in [10] to dg bifunctors. Concretely, if $A, B$ and $C$ are dg algebras with enough idempotents and $F:(D g-A) \otimes(D g-C) \longrightarrow D g-B$ is a dg functor which preserves contractibility on both variables, then one defines

$$
\mathbb{L} F: \mathcal{D}(A) \otimes \mathcal{D}(C) \stackrel{\Pi_{A} \otimes \Pi_{C}}{\longrightarrow} \mathcal{H}(A) \otimes \mathcal{H}(C) \stackrel{H^{0} F}{\longrightarrow} \mathcal{H}(B) \stackrel{q_{B}}{\longrightarrow} \mathcal{D}(B),
$$

and

$$
\mathbb{R} F: \mathcal{D}(A) \otimes \mathcal{D}(C) \stackrel{\Upsilon \otimes \Upsilon_{C}}{\longrightarrow} \mathcal{H}(A) \otimes \mathcal{H}(C) \stackrel{H^{0} F}{\longrightarrow} \mathcal{H}(B) \stackrel{q_{B}}{\longrightarrow} \mathcal{D}(B)
$$


When $F$ is contravariant on the first variable, i.e. when $F:(D g-A)^{o p} \otimes(D g-C) \longrightarrow D g-B$ is a dg functor, one also defines

$$
\mathbb{R} F: \mathcal{D}(A)^{o p} \otimes \mathcal{D}(C) \stackrel{\Pi_{A}^{o} \otimes \Upsilon_{C}}{\longrightarrow} \mathcal{H}(A) \otimes \mathcal{H}(C) \stackrel{H^{0} F}{\longrightarrow} \mathcal{H}(B) \stackrel{q_{B}}{\longrightarrow} \mathcal{D}(B) .
$$

The point is that, under suitable conditions (see [10, Proposition 7.17] for details), these later bifunctors are triangulated on each variable.

1.5. Derived Hom and $\otimes$ functors. Given dg algebras with enough idempotents $A, B$ and $C$ and $\mathrm{dg}$ bimodules ${ }_{C} M_{A},{ }_{B} X_{A}$ and ${ }_{C} U_{B}$, the $\mathrm{dg} K$-modules $\mathrm{HOM}_{A}(M, X)$ and $U \otimes_{B} X$ have canonical structures of $\operatorname{dg} B-C$-bimodule and $\operatorname{dg} C-A$-bimodule, respectively, but the first one is non-unitary. This forces to define the 'unitarization'

$$
\overline{\mathrm{HOM}}_{A}(M, X):=B \mathrm{HOM}_{A}(M, X) C,
$$

which is then a (now unitary!) $\operatorname{dg} B-C$-bimodule. It is proved in [10] that the assignments $(M, X) \rightsquigarrow \overline{\operatorname{HOM}}_{A}(M, X)$ and $(U, X) \rightsquigarrow U \otimes_{B} X$ are the definition on objects of dg functors

$$
\begin{aligned}
\overline{\mathrm{HOM}}_{A}(?, ?):(C-D g-A)^{o p} \otimes(B-D g-A) & \longrightarrow B-D g-C \\
? \otimes_{B} ?:(C-D g-B) \otimes(B-D g-A) & \longrightarrow C-D g-A .
\end{aligned}
$$

One then puts

$$
\begin{aligned}
& ? \otimes_{B}^{\mathbb{L}} X:=\mathbb{L}\left(? \otimes_{B} X\right): \mathcal{D}\left(B \otimes C^{o p}\right) \quad \longrightarrow \mathcal{D}\left(A \otimes C^{o p}\right), \\
& U \otimes_{B}^{\mathbb{L}} ?:=\mathbb{L}\left(U \otimes_{B} ?\right): \mathcal{D}\left(A \otimes B^{o p}\right) \longrightarrow \mathcal{D}\left(A \otimes C^{o p}\right), \\
& \mathbb{R H o m}_{A}(M, ?):=\mathbb{R}\left(\overline{\mathrm{HOM}}_{A}(M, ?)\right): \mathcal{D}\left(A \otimes B^{o p}\right) \longrightarrow \mathcal{D}\left(C \otimes B^{o p}\right) \\
& \mathbb{R H o m}_{A}(?, X):=\mathbb{R}\left(\overline{\operatorname{HOM}}_{A}(?, X)\right): \mathcal{D}\left(A \otimes C^{o p}\right)^{o p} \longrightarrow \mathcal{D}\left(C \otimes B^{o p}\right) \text {. }
\end{aligned}
$$

By [10, Theorems 9.1 and 9.5], the pairs

$$
\left(? \otimes_{B} X: C-D g-A \longrightarrow C-D g-A, \overline{\operatorname{HOM}}_{A}(X, ?): C-D g-A \longrightarrow C-D g-B\right)
$$

and

$\left(\overline{\mathrm{HOM}}_{B^{o p}}(?, X)^{o}: B-D g-C \rightarrow(C-D g-A)^{o p}, \overline{\mathrm{HOM}}_{A}(?, X):(C-D g-A)^{o p} \rightarrow B-D g-C\right)$

are $\mathrm{dg}$ adjunctions and, hence, we get adjunctions of triangulated functors

$$
\left(? \otimes_{B}^{\mathbb{L}} X: \mathcal{D}\left(B \otimes C^{o p}\right) \longrightarrow \mathcal{D}\left(A \otimes C^{o p}\right), \mathbb{R H o m}_{A}(X, ?): \mathcal{D}\left(A \otimes C^{o p}\right) \longrightarrow \mathcal{D}\left(B \otimes C^{o p}\right)\right)
$$

and

$\left.\left.\left(\mathbb{R H o m}_{B^{o p}}(?, X)^{o}: \mathcal{D}\left(C \otimes B^{o p}\right) \longrightarrow \mathcal{D}\left(A \otimes C^{o p}\right)\right)^{o p}, \mathbb{R H o m}_{A}(?, X): \mathcal{D}\left(A \otimes C^{o p}\right)\right)^{o p} \longrightarrow \mathcal{D}\left(C \otimes B^{o p}\right)\right)$.

By the previous paragraph, one also defines

$$
\mathbb{R H O M}_{A}(?, ?):=\mathbb{R}\left(\overline{\mathrm{HOM}}_{A}(?, ?)\right): \mathcal{D}\left(A \otimes C^{o p}\right)^{o p} \otimes \mathcal{D}\left(A \otimes B^{o p}\right) \longrightarrow \mathcal{D}\left(C \otimes B^{o p}\right),
$$

which is then a functor which is triangulated in each variable. Moreover, precise conditions are given in 10, Corollary 9.7] to have a natural isomorphisms triangulated functors $\mathbb{R H O M}_{A}(M, ?) \cong \mathbb{R} \operatorname{Hom}_{A}(M, ?)$ and $\mathbb{R H O M}_{A}(?, X) \cong \mathbb{R} \operatorname{Hom}_{A}(?, X)$. In particular, by taking $C=K$ in [10, Corollary 9.7] and its proof, one gets the following consequence, which will frequently be used in Section 4 .

Proposition 2. Let $A$ and $B$ be $d g$ algebras with enough idempotents and let

$$
\mathbb{R} H O M_{A}(?, ?):=\mathbb{R}\left(\overline{H O M}_{A}(?, ?)\right): \mathcal{D}\left(A^{o p}\right) \otimes \mathcal{D}\left(A \otimes B^{o p}\right) \longrightarrow \mathcal{D}\left(B^{o p}\right)
$$

be the associated bi-triangulated functor. There are natural isomorphisms of triangulated functors, for all $d g B-A$-bimodules $X$ and all right $d g$ A-modules $M$ :

(1) $\mathbb{R} H O M_{A}(?, X) \cong \mathbb{R} H_{A} m_{A}(?, X): \mathcal{D}(A)^{o p} \longrightarrow \mathcal{D}\left(B^{o p}\right)$.

(2) $\mathbb{R} H O M_{A}(M, ?) \cong \mathbb{R} H_{o m}\left(\Pi_{A}(M), ?\right): \mathcal{D}\left(A \otimes B^{o p}\right) \longrightarrow \mathcal{D}\left(B^{o p}\right)$. 
On the other hand, when $X={ }_{A} A_{A}$ is the regular dg bimodule associated to the $\mathrm{dg}$ algebra with enough idempotents $A$, one has that the adjunction $\left(\mathbb{R} \operatorname{Hom}_{A^{o p}}(?, A)^{o}: \mathcal{D}\left(A^{o p}\right) \longrightarrow\right.$ $\left.\mathcal{D}(A)^{o p}, \mathbb{R H o m}_{A}(?, A): \mathcal{D}(A)^{o p} \longrightarrow \mathcal{D}\left(A^{o p}\right)\right)$ gives by restriction quasi-inverse dualities $\operatorname{per}\left(A^{o p}\right) \stackrel{\cong o}{\longrightarrow} \operatorname{per}(A)$, where $\operatorname{per}(A)=\mathcal{D}^{c}(A)$ (resp. $\left.\operatorname{per}\left(A^{o p}\right)=\mathcal{D}^{c}\left(A^{o p}\right)\right)$ is the right (resp. left) perfect derived category of $A$, i.e. the full subcategory of $\mathcal{D}(A)\left(\operatorname{resp} . \mathcal{D}\left(A^{o p}\right)\right)$ consisting of the compact objects. It is this duality what will allow us to pass from the left version of degeneration to the right version, and vice versa, in the proof of Theorem 1 under hypothesis (b).

\section{REVIEW OF DEGENERATION IN TRIANGULATED CATEGORIES}

We start to recall from [4, 11] a few facts on the concept of degeneration of objects in triangulated categories.

2.1. The module case. We first recall a classical result due to Zwara and Riedtmann [9, 20]. Let $A$ be a $k$-algebra over an algebraically closed field $k$ and two finite dimensional $A$-modules $M$ and $N$. Then $N$ belongs to the closure of the orbit of $M$ if and only if there is a finite dimensional $A$-module $Z_{r}$ and a short exact sequence

$$
0 \longrightarrow N \longrightarrow M \oplus Z_{r} \stackrel{\left(u_{r} \quad v_{r}\right)}{\longrightarrow} Z_{r} \longrightarrow 0,
$$

where $v_{r}$ is a nilpotent endomorphism of $Z_{r}$. We say in this case that $M$ degenerates to $N$. Zwara shows in [19, Theorem 5] that $M$ degenerates to $N$ if and only if there is a finite dimensional $A$-module $Z_{\ell}$ of $\mathcal{T}$ and an exact sequence triangle

$$
0 \longrightarrow Z_{\ell} \stackrel{\left(\begin{array}{l}
v_{\ell} \\
u_{\ell}
\end{array}\right)}{\longrightarrow} Z_{\ell} \oplus M \longrightarrow N \longrightarrow 0,
$$

where $v_{\ell}$ is a nilpotent endomorphism of $Z_{\ell}$.

2.2. Generalising degeneration to triangulated categories. In our previous work [4, 11, and independently by work of Yoshino [15] in the case of stable categories of maximal Cohen-Macaulay modules of local Gorenstein algebras, the concept of degeneration for modules was generalised to triangulated categories. Yoshino discovered in particular the importance of the hypothesis that the induced endomorphism of $Z_{\ell}$ (resp. $Z_{r}$ ) is nilpotent.

Definition 3. For two objects $M$ and $N$ of a triangulated category $\mathcal{T}$ we say that $M$ degenerates to $N$ in the triangle sense and write $M \leq_{\Delta+n i l} N$ if and only if there is an object $Z_{r}$ of $\mathcal{T}$ and a distinguished triangle in $\mathcal{T}$

$$
Z_{\ell} \stackrel{\left(\begin{array}{l}
u_{\ell} \\
v_{\ell}
\end{array}\right)}{\longrightarrow} M \oplus Z_{\ell} \longrightarrow N \longrightarrow Z_{\ell}[1],
$$

where $v_{\ell}$ is a nilpotent endomorphism of $Z_{\ell}$.

The main purpose of [11] was to define a geometric notion of degeneration along the lines of [15], and to prove that this notion is equivalent with the notion of degeneration in the triangle sense. More precisely we gave the following definition.

Definition 4 . Let $K$ be a commutative ring and let $\mathcal{C}_{K}^{\circ}$ be a $K$-linear triangulated category with split idempotents.

A degeneration data for $\mathcal{C}_{K}^{\circ}$ is given by

- a triangulated category $\mathcal{C}_{K}$ with split idempotents and a fully faithful embedding $\mathcal{C}_{K}^{\circ} \longrightarrow \mathcal{C}_{K}$

- a triangulated category $\mathcal{C}_{V}$ with split idempotents and a full triangulated subcategory $\mathcal{C}_{V}^{\circ}$ 
- triangulated functors $\uparrow_{K}^{V}: \mathcal{C}_{K} \longrightarrow \mathcal{C}_{V}$ and $\Phi: \mathcal{C}_{V}^{\circ} \rightarrow \mathcal{C}_{K}$, so that $\left(\mathcal{C}_{K}^{\circ}\right) \uparrow{ }_{K} \subseteq \mathcal{C}_{V}^{\circ}$, when we view $\mathcal{C}_{K}^{\circ}$ as a full subcategory of $\mathcal{C}_{K}$,

- a natural transformation $\operatorname{id}_{\mathcal{C}_{V}} \stackrel{t}{\longrightarrow} \operatorname{id}_{\mathcal{C}_{V}}$ of triangulated functors

These triangulated categories and functors should satisfy the following axioms:

(1) For each object $M$ of $\mathcal{C}_{K}^{\circ}$ the morphism $\Phi\left(M \uparrow_{K}^{V}\right) \stackrel{\Phi\left(t_{M \uparrow \uparrow_{K}}\right)}{\longrightarrow} \Phi\left(M \uparrow_{K}^{V}\right)$ is a split monomorphism in $\mathcal{C}_{K}$.

(2) For all objects $M$ of $\mathcal{C}_{K}^{\circ}$ we get $\Phi\left(\operatorname{cone}\left(t_{M \uparrow V}\right)\right) \simeq M$.

Degeneration is then given by the following concept.

Definition 5. Given two objects $M$ and $N$ of $\mathcal{C}_{K}^{\circ}$ we say that $M$ degenerates to $N$ in the categorical sense if there is a degeneration data for $\mathcal{C}_{K}^{\circ}$ and an object $Q$ of $\mathcal{C}_{V}^{\circ}$ such that

$$
p(Q) \simeq p\left(M \uparrow_{k}^{V}\right) \text { in } \mathcal{C}_{V}^{\circ}\left[t^{-1}\right] \text { and } \Phi\left(\operatorname{cone}\left(t_{Q}\right)\right) \simeq N,
$$

where $p: \mathcal{C}_{V}^{\circ} \longrightarrow \mathcal{C}_{V}^{\circ}\left[t^{-1}\right]$ is the canonical functor. In this case we write $M \leq_{c d e g} N$.

Example 6. Yoshino observed that in a triangulated category $\mathcal{T}$ for all objects $X$ we get $0 \leq \Delta+n i l=X \oplus X[1]$. Indeed, $X \rightarrow 0 \rightarrow X[1] \stackrel{i d}{\rightarrow} X[1]$ and $0 \rightarrow X \stackrel{i d}{\rightarrow} X \rightarrow 0$ are distinguished triangles for each object $X$. Hence their direct sum $X \stackrel{0}{\rightarrow} X \oplus 0 \rightarrow X \oplus X[1] \rightarrow X[1]$ is a distinguished triangle as well. Taking $Z=X$ we get the result (in the left version of $\left.\leq_{\Delta+n i l}\right)$.

Now, what about the degeneration data interpretation, which is equivalent to the triangle version in important cases? Then there is an object $Q$ in some triangulated category $\mathcal{C}_{V}^{\circ}$ and an element $t$ in its centre such that $\Phi\left(\operatorname{cone}\left(t_{Q}\right)\right) \simeq X \oplus X[1]$ and $p(Q) \simeq 0$ in $\mathcal{C}_{V}^{\circ}\left[t^{-1}\right]$, where $p$ is the localisation functor. The latter isomorphism is equivalent to the fact that $t_{Q}$ is nilpotent on $Q$. Hence we cannot assume, and actually do not assume, that $Q$ is $t$-flat, as Yoshino does in the case of modules [14.

2.3. When triangle degeneration is the same as categorical degeneration. The main result of [11] is the following.

Theorem 7. Let $K$ be a commutative ring and let $\mathcal{C}_{K}^{\circ}$ be a triangulated $K$-category with split idempotents. If $M$ and $N$ are objects of $\mathcal{C}_{K}^{\circ}$, then $M \leq_{\text {cdeg }} N \Rightarrow M \leq_{\Delta+n i l} N$. When $\mathcal{C}_{K}^{\circ}$ is equivalent to the category of compact objects of a compactly generated algebraic triangulated $K$-category, the converse is also true.

In order to prove that $\leq_{\Delta+n i l}$ implies $\leq_{c d e g}$ for the category of compact objects of a compactly generated algebraic triangulated $K$-category, we need to construct a degeneration data. By a result of Keller [5], we know that $\mathcal{T}$ is equivalent to the category $\mathcal{D}^{c}(\mathcal{A})$ of compact objects of the derived category $\mathcal{D}(\mathcal{A})$ of some small dg-category $\mathcal{A}$. We construct then the degeneration data for $\mathcal{C}_{K}^{o}=\mathcal{D}^{c}(\mathcal{A})$ very explicitly, constructing a dg category $\mathcal{A}[[T]]$ from $\mathcal{A}$ and our proof of Theorem 1 under hypothesis (b), which is given in Section 4 , uses this construction. More precisely, recall from the proof of [11, Proposition 9] that if $\mathcal{A}$ is a small dg category, then, considering a variable $T$, one can form a new dg category $\mathcal{A}[[T]]$ with the same set of objects as $\mathcal{A}$ and where one defines

$$
\operatorname{Hom}_{\mathcal{A}[[T]]}^{n}\left(A, A^{\prime}\right)=\left\{\sum_{k \in \mathbb{N}} \alpha_{k} T^{k}: \alpha_{k} \in \operatorname{Hom}_{\mathcal{A}}^{n}\left(A, A^{\prime}\right) \text {, for all } k \in \mathbb{N}\right\} .
$$

Moreover, one gets a canonical functor

$$
? \hat{\otimes} V: \mathcal{C}(\mathcal{A}) \longrightarrow \mathcal{C}(\mathcal{A}[[T]])
$$

which takes a right $\operatorname{dg} \mathcal{A}$-module $M$ to the right $\operatorname{dg} \mathcal{A}[[T]]$-module $M[[T]]: \mathcal{A}^{\text {op }} \longrightarrow \mathcal{C}_{d g} K$ acting on objects as $M[[T]]^{n}(A)=M^{n}(A)[[T]]$, for all $n \in \mathbb{Z}$ and all $A \in \mathcal{A}$. The degeneration data for $\mathcal{C}_{K}^{o}$ is then given by taking $\mathcal{C}_{K}=\mathcal{D}(\mathcal{A})$, with the corresponding inclusion functor as $\mathcal{C}_{K}^{o} \longrightarrow \mathcal{C}_{K}, \mathcal{C}_{V}=\mathcal{D}(\mathcal{A}[[T]]), \mathcal{C}_{V}^{o}=\mathcal{D}^{c}(\mathcal{A}[[T]])$, the triangulated version of 
$? \hat{\otimes} V$ as functor $\uparrow_{K}^{V}: \mathcal{C}_{K}=\mathcal{D}(\mathcal{A}) \longrightarrow \mathcal{D}(\mathcal{A}[[T]])=\mathcal{C}_{V}$, the restriction of scalars functor as $\phi: \mathcal{C}_{V}^{o}=\mathcal{D}^{c}(\mathcal{A}[[T]]) \longrightarrow \mathcal{D}(\mathcal{A})$ and the natural transformation $t: i d_{\mathcal{C}_{V}} \longrightarrow i d_{\mathcal{C}_{V}}$ is defined by the maps $t_{Q}: Q \longrightarrow Q$, for each $\operatorname{dg} \mathcal{A}[[T]]$-module $Q$, given by multiplication by $T$.

We take the opportunity to mention that in [11] we forgot to mention the grading, although it was implicit in all of the proofs because, when dealing with dg categories and dg modules, normally one only uses homogeneous elements. However, a potential reader of [11] might think that there is an error in the definition of $\mathcal{A}[[T]]$ and of $M[[T]]$ for, as it is written, they are not a graded category or a graded module. The proof of [11] does need not to be modified.

\section{The CASE OF triangulated CATEGORIES WHOSE OBJECTS HAVE ARTINIAN ENDOMORPHISM ALGEBRAS}

In this section we shall prove the first part of the theorem. We will mimic Zwara's proof to give the analogous statement for triangle degeneration.

3.1. Generalities on homotopy cartesian squares in triangulated categories. As in [19] we first need some preparation. Throughout this section let $\mathcal{T}$ be a triangulated $K$-category for a commutative base ring $K$. The crucial concept is that of a homotopy cartesian square. Recall from [8, 1.4] that a commutative diagram

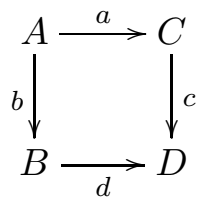

is homotopy cartesian if there is a map $e: D \rightarrow A[1]$ such that

$$
A \stackrel{\left(\begin{array}{l}
b \\
a
\end{array}\right)}{\longrightarrow} B \oplus C \stackrel{(-c, d)}{\longrightarrow} D \stackrel{e}{\longrightarrow} A[1]
$$

is a distinguished triangle. In the rest of this section, denote by $C_{f}$ the cone of any morphism $f$ in $\mathcal{T}$. By [8, Lemma 1.4.3], if a commutative square

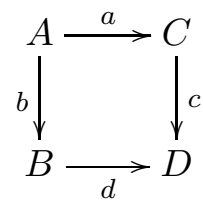

is homotopy cartesian, then there is an isomorphism $\sigma: C_{b} \stackrel{\cong}{\longrightarrow} C_{d}$ such that

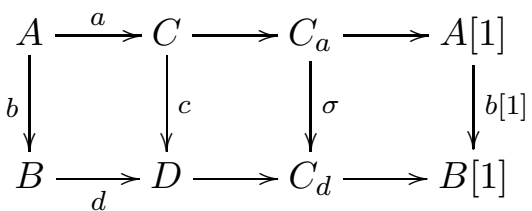

is commutative with rows being distinguished triangles. [8, Lemma 1.4.4] gives a partial converse. If

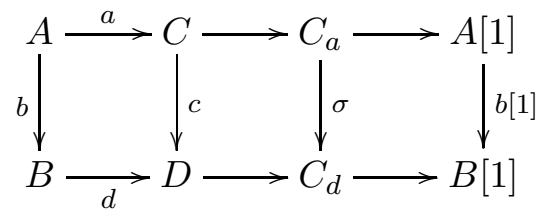


is commutative, where the rows are distinguished triangles and $\sigma$ is an isomorphism, then there is a possible different $b^{\prime}: A \rightarrow B$ such that still

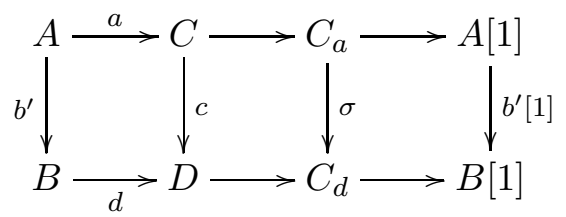

is commutative and furthermore

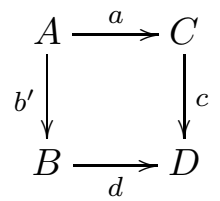

is homotopy cartesian. We may alternatively modify $c$ instead of $b$.

This problem has the annoying consequence that if we have two homotopy cartesian squares

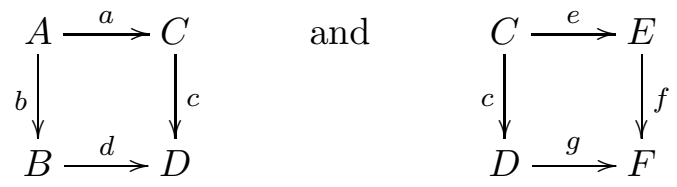

then there is $b^{\prime}$ and $f^{\prime}$ fitting in certain morphisms of triangles, such that

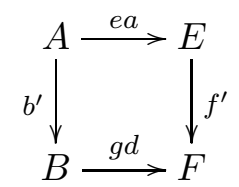

is a homotopy cartesian square. We would like to be able to assume that $b^{\prime}=b$ and $f^{\prime}=f$. However, we do not know if this is true. Nevertheless, we prove a weaker statement which satisfy our needs.

Lemma 8. Let

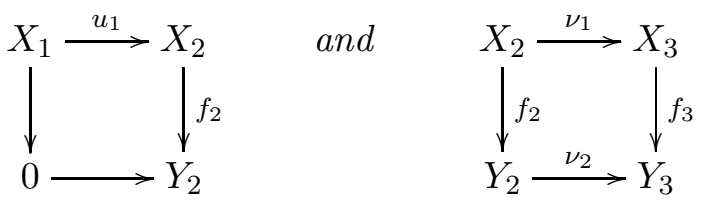

be homotopy cartesian squares. Then

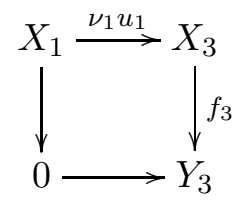

is a homotopy cartesian square.

Proof. We first apply the octahedron axiom to the composition $\nu_{1} u_{1}$. We hence obtain a morphism $v: X_{3} \rightarrow C_{\nu_{1} u_{1}}$, a morphism $\omega: Y_{2} \rightarrow C_{\nu_{1} u_{1}}$, a morphism $C_{\nu_{1} u_{1}} \rightarrow C_{\nu_{1}}$, and 

$C_{\nu_{1}} \rightarrow Y_{2}[1]$ as indicated below,

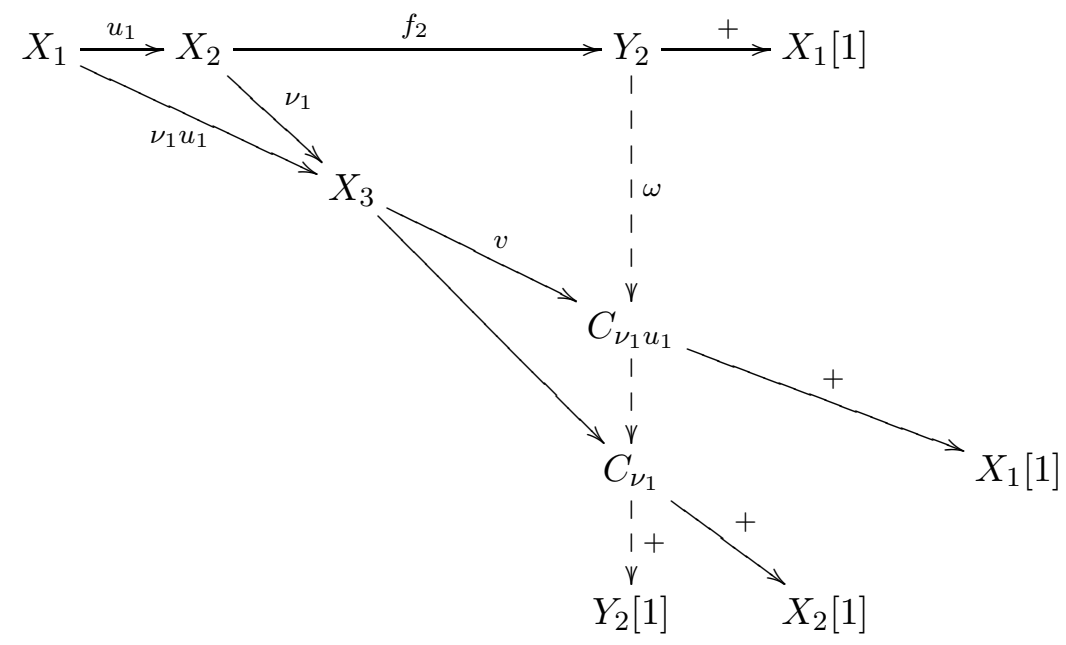

such that all straight sequences represent distinguished triangles, and such that the diagram is commutative. In particular, $v \nu_{1}=\omega f_{2}$. Neeman's interpretation of the octahedral axiom [8, Proposition 1.4.6] implies that we may choose $v$ and $\omega$ such that

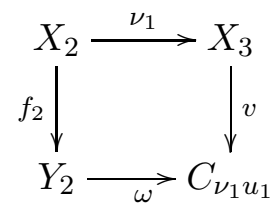

is a homotopy cartesian square. Since

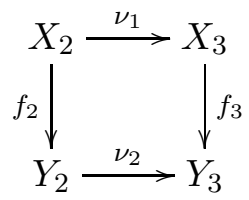

is homotopy cartesian by hypothesis, there is an isomorphism $\varphi: C_{\nu_{1} u_{1}} \stackrel{\cong}{\longrightarrow} Y_{3}$ such that

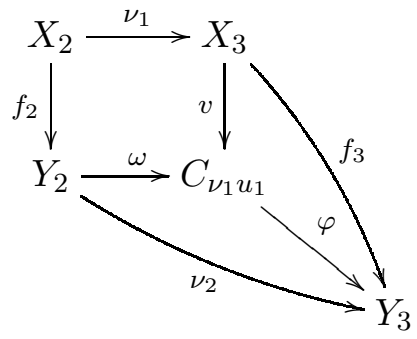

is commutative. This then shows that

$$
X_{1} \stackrel{\nu_{1} u_{1}}{\longrightarrow} X_{3} \stackrel{f_{3}}{\longrightarrow} Y_{3} \longrightarrow X_{1}[1]
$$

is a distinguished triangle, and hence

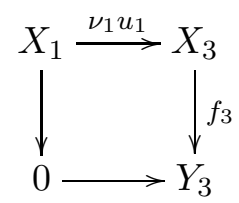

is a homotopy cartesian square as claimed.

A partial converse is true in general however. 
Lemma 9. Let

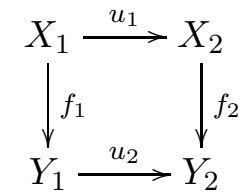

and

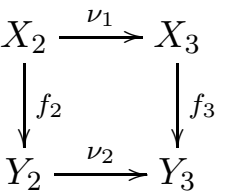

be commutative diagrams. If the first square and

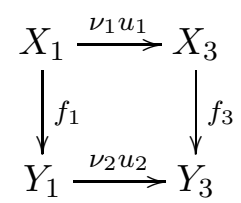

are homotopy cartesian squares, then there is a morphism $\hat{\nu}_{2}$ with $\nu_{2} u_{2}=\hat{\nu}_{2} u_{2}$ such that

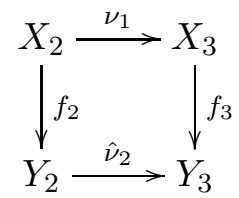

is a homotopy cartesian square.

Proof. We supposed that
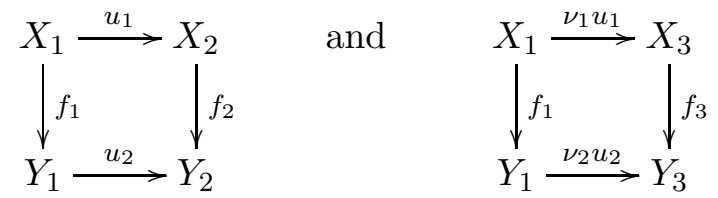

are homotopy cartesian squares. Hence we get distinguished triangles

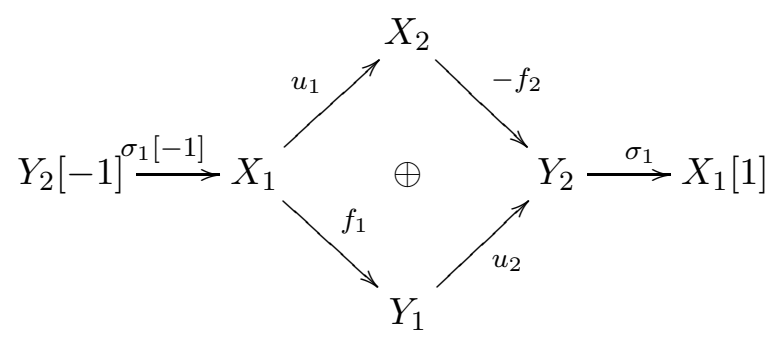

and

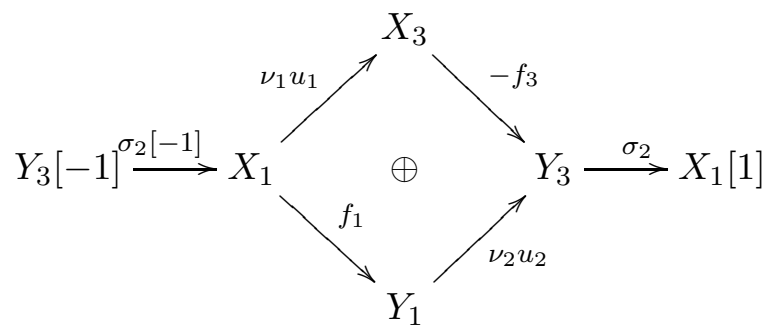

We further get a morphism of distinguished triangles by the fact that the second comes from a square which factors through the first one. 


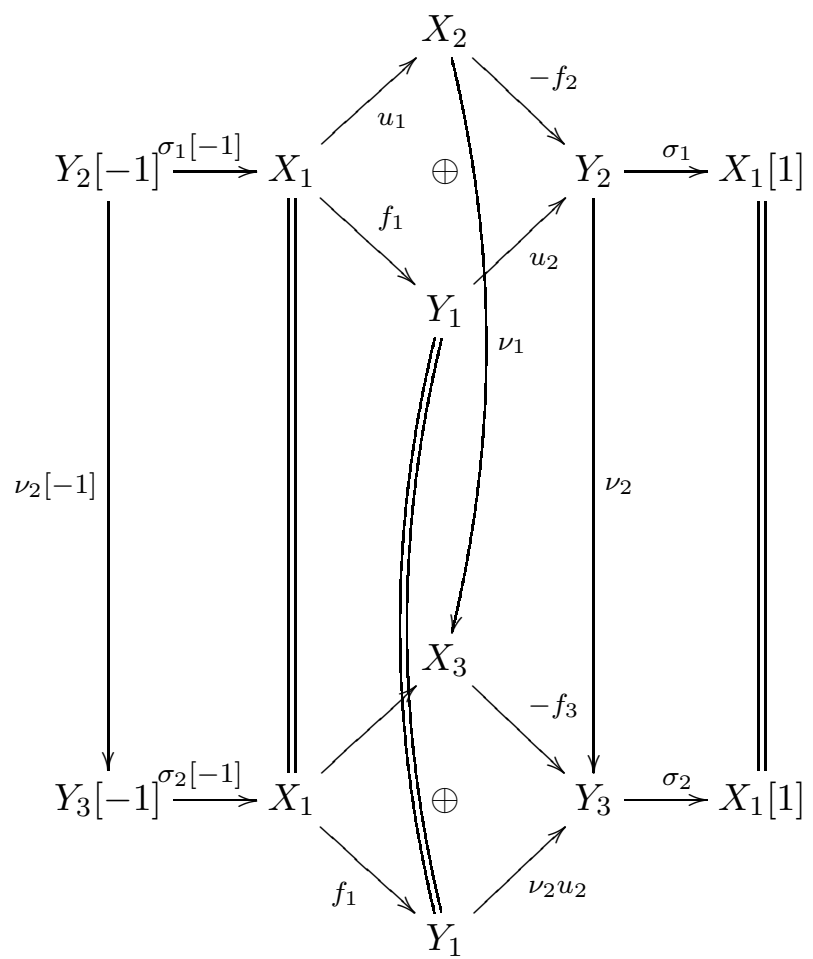

By Neeman's axiom TR4' we maybe need to modify $\nu_{2}$ to another map $\hat{\nu}_{2}$, forming still a map of distinguished triangles, so that the cone of this commutative diagram is a distinguished triangle.

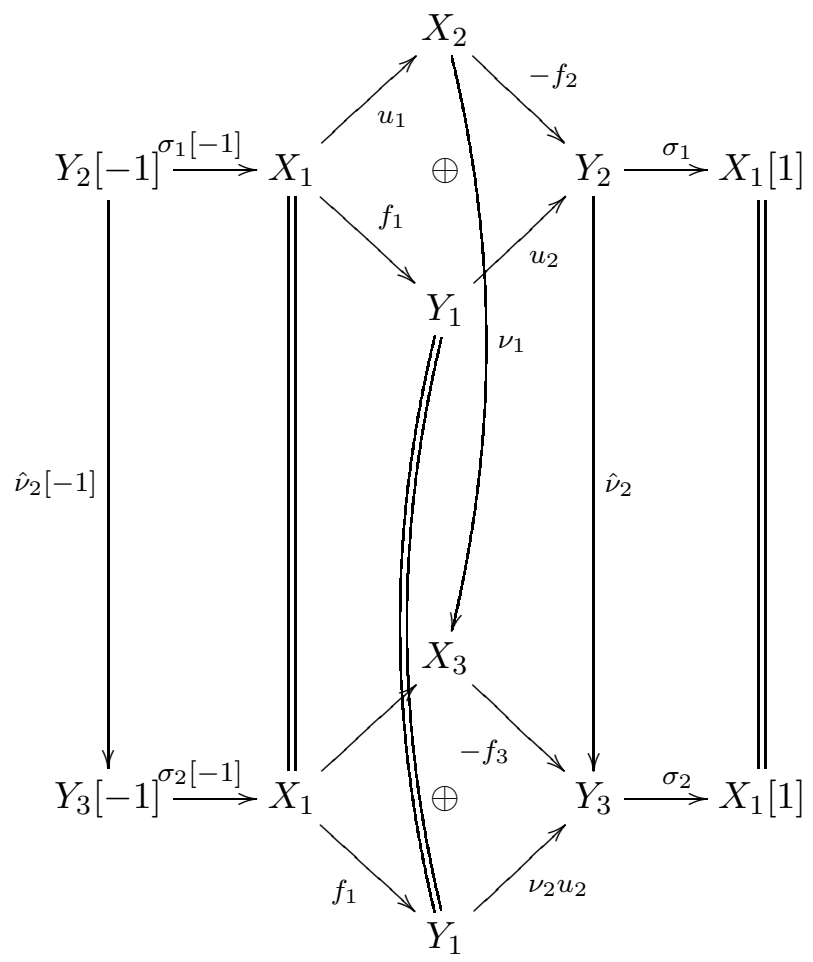

This implies in particular that $\nu_{2} u_{2}=\hat{\nu}_{2} u_{2}$. The cone of this has a direct factor isomorphic to 


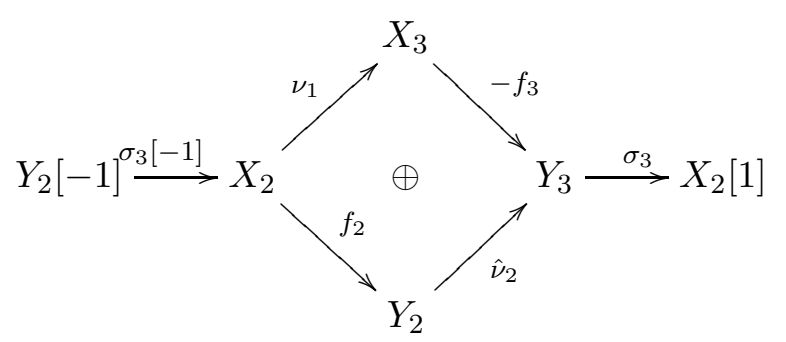

which is therefore a distinguished triangle. This proves the statement.

3.2. Triangle degeneration is symmetric with respect to left or right existence of the object $Z$. Recall the following result of Xiao-Wu Chen, Yu Ye and Phu Zhang.

Proposition 10. [2, Theorem A.1] An additive category $\mathcal{C}$ is Krull-Schmidt if and only if any idempotent splits, and the endomorphism ring of any object of $\mathcal{C}$ is semiperfect.

Our main result of this section now is the following.

Theorem 11. Let $K$ be a commutative ring and let $\mathcal{T}$ be a $K$-linear triangulated category with split idempotents and such that the endomorphism ring of each object is artinian, and let $M$ and $N$ be two objects. Then there is an object $Z_{r}$ and a distinguished triangle

$$
N \rightarrow M \oplus Z_{r} \stackrel{\left(u_{r}, v_{r}\right)}{\longrightarrow} Z_{r} \longrightarrow N[1]
$$

(with nilpotent $v_{r}$ ) if and only if there is an object $Z_{\ell}$ and a distinguished triangle

$$
Z_{\ell} \stackrel{\left(\begin{array}{l}
u_{\ell} \\
v_{\ell}
\end{array}\right)}{\longrightarrow} M \oplus Z_{\ell} \longrightarrow N \longrightarrow Z_{\ell}[1]
$$

(with nilpotent $\left.v_{\ell}\right)$.

Remark 12. The hypothesis that each object in $\mathcal{T}$ has artinian endomorphism ring implies that $\mathcal{T}$ is Krull-Schmidt, and moreover that we get Fitting's lemma for $\mathcal{T}$. In particular, and endomorphism $\nu: Z \rightarrow Z$ can be decomposed into

$$
\nu=\left(\begin{array}{cc}
\nu^{\prime} & 0 \\
0 & \nu^{\prime \prime}
\end{array}\right): Z=Z^{\prime} \oplus Z^{\prime \prime} \rightarrow Z^{\prime} \oplus Z^{\prime \prime}=Z
$$

and such that $\nu^{\prime}$ is an automorphism and $\nu^{\prime \prime}$ is nilpotent. Splitting off the trivial triangle $Z_{\ell}^{\prime} \stackrel{\nu^{\prime}}{\longrightarrow} Z_{\ell}^{\prime} \rightarrow 0 \rightarrow Z_{\ell}^{\prime}[1]$, respectively $0 \rightarrow Z_{r}^{\prime} \stackrel{\nu^{\prime}}{\longrightarrow} Z_{r}^{\prime} \rightarrow 0$ [1] we may hence assume that $v_{\ell}$ and $v_{r}$ are nilpotent.

Proof. (of Theorem 11) Set $N_{1}:=N$. Let $Z_{\ell}$ be an object and let

$$
Z_{\ell} \stackrel{\left(\begin{array}{l}
u_{\ell} \\
v_{\ell}
\end{array}\right)}{\longrightarrow} M \oplus Z_{\ell} \stackrel{\left(\nu_{1}, h_{1}\right)}{\longrightarrow} N_{1} \stackrel{\rho_{0}}{\longrightarrow} Z_{\ell}[1]
$$

be a distinguished triangle with nilpotent endomorphism $v_{\ell}$. Then we form the homotopy pushout (cf [8, Lemma 1.4.3 and 1.4.4])

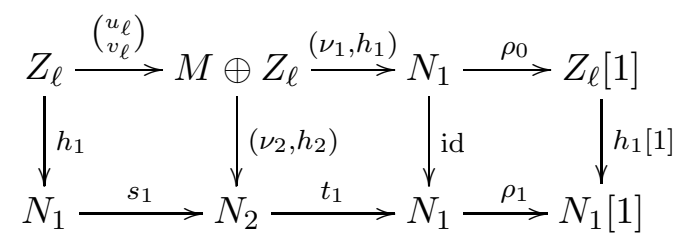


We get $h_{1}=t_{1} h_{2}$ from the commutativity of the middle square and $h_{1}[1] \rho_{0}=\rho_{1}$ from the right most square. We form the homotopy pushout

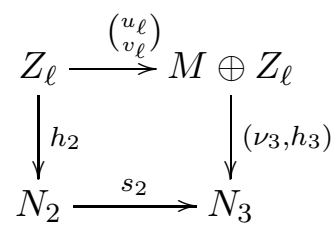

and obtain a commutative diagram with homotopy pushouts on the front face and on the back face. Denote for short $\hat{v}:=\left(\begin{array}{l}v_{\ell} \\ u_{\ell}\end{array}\right)$.

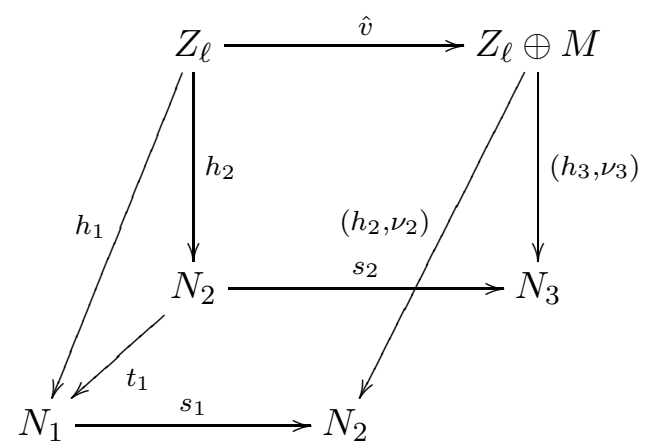

Since the back face is a homotopy pushout, and since the diagram

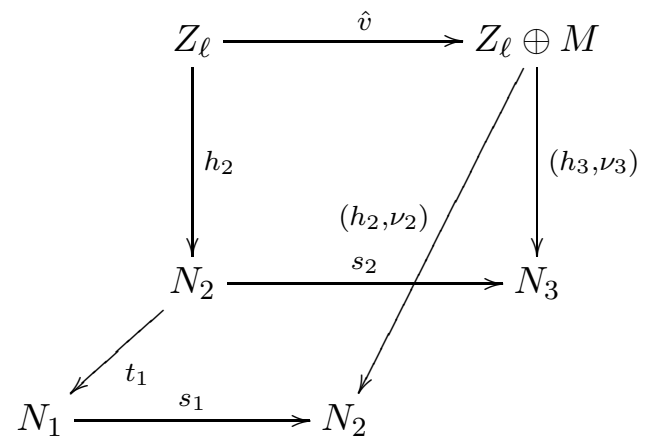

is commutative, there is a non-unique map $t_{2}: N_{3} \rightarrow N_{2}$ making the diagram

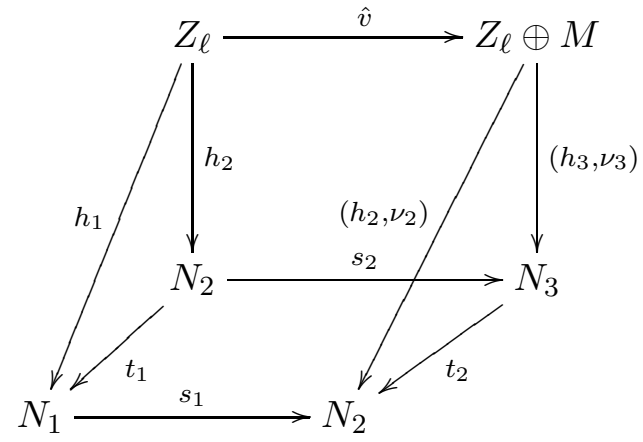

commutative. Lemma 9 then allows to modify $t_{2}$ such that the bottom face of the diagram is homotopy cartesian, such that all already shown commutativity properties still hold, and such that the above diagram with modified $t_{2}$ is still commutative. Here, in order to simplify the notation, we denote the modified $t_{2}$ again by $t_{2}$.

We proceed now by induction on the degree $n$. Suppose we have a commutative diagram with rows being distinguished triangles and whose square faces are homotopy cartesian 
squares

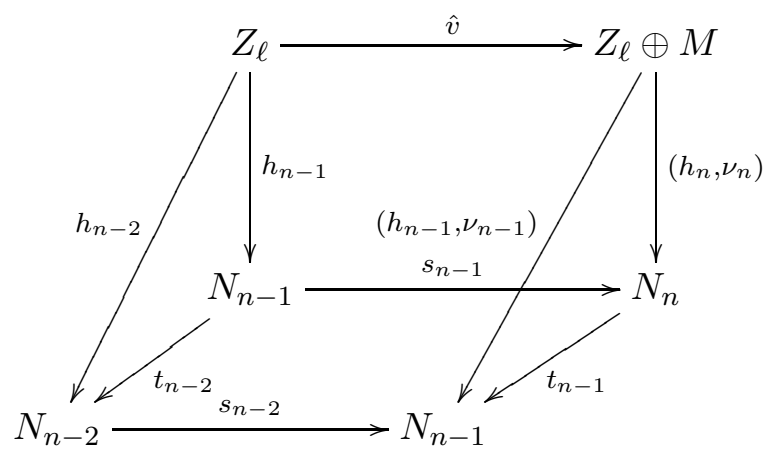

We form the homotopy pushout (on the back face of the diagram defining $N_{n+1}, h_{n+1}$, $s_{n}$ and $\left.\nu_{n+1}\right)$

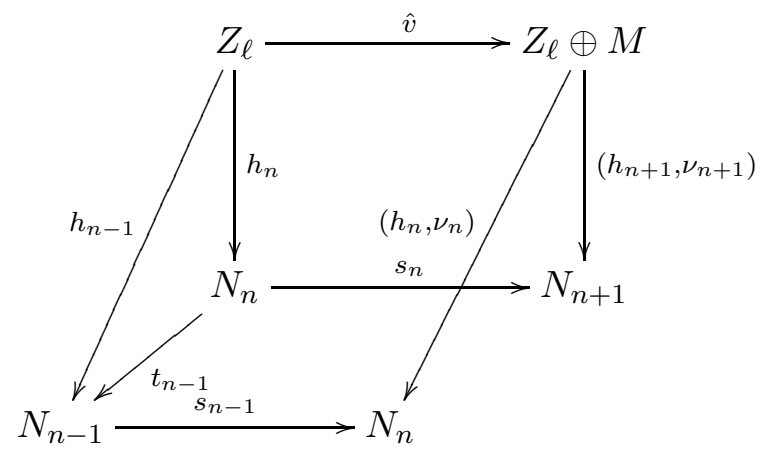

Since the diagram

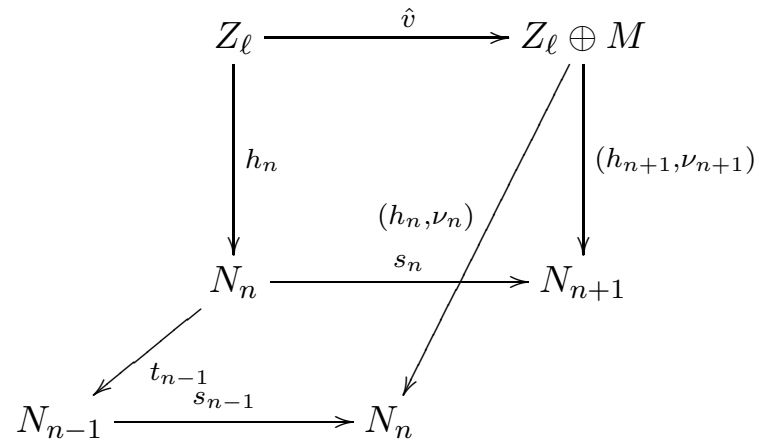

is commutative, there is a morphism $t_{n}: N_{n+1} \rightarrow N_{n}$ making the diagram

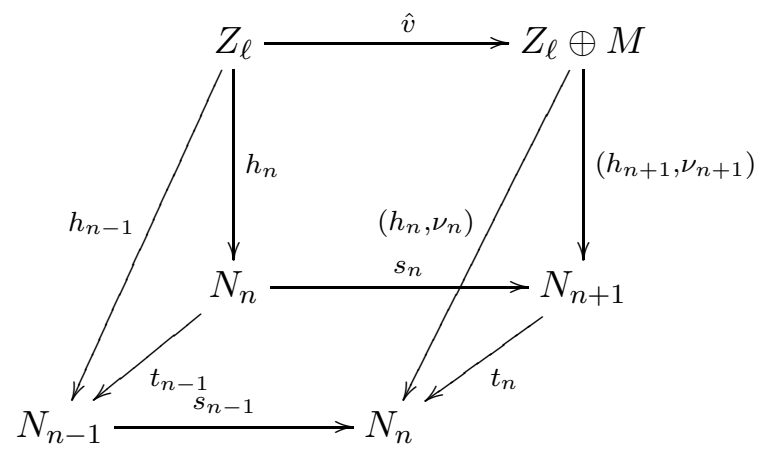

commutative. By Lemma 9 we may modify $t_{n}$ without changing commutativity of what is already shown (we denote the modified $t_{n}$ again by $t_{n}$ ), such that the bottom face of the diagram is a homotopy pushout and such the above diagram is still commutative.

We now continue as in the proof of [19, Theorem 2.3]. We define $\omega_{1}:=\nu_{1}$ and

$$
\omega_{j+1}:=\left(\nu_{j+1}, s_{j} \omega_{j}\right) \in \operatorname{Hom}_{\mathcal{T}}\left(M \oplus M^{j}, N_{j+1}\right)=\operatorname{Hom}_{\mathcal{T}}\left(M^{j+1}, N_{j+1}\right)
$$


for all $j \geq 1$.

In the diagram

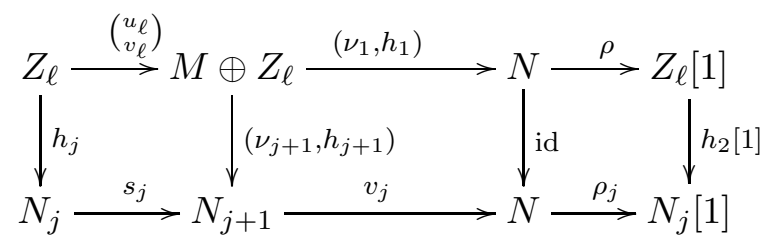

the left square is a homotopy cartesian square. Therefore we get a distinguished triangle

$$
Z_{\ell} \stackrel{\left(\begin{array}{c}
u_{\ell} \\
v_{\ell} \\
h_{j}
\end{array}\right)}{\longrightarrow} M \oplus Z_{\ell} \oplus N_{j} \stackrel{\left(\nu_{j+1}, h_{j+1},-s_{j}\right)}{\longrightarrow} N_{j+1} \stackrel{\sigma_{j}}{\longrightarrow} Z_{\ell}[1]
$$

Since

$$
M^{j} \stackrel{\mathrm{id}}{\longrightarrow} M^{j} \longrightarrow 0 \longrightarrow M^{j}[1]
$$

is a distinguished triangle, also the direct sum of these two distinguished triangles

$$
M^{j} \oplus Z_{\ell} \stackrel{\left(\begin{array}{cc}
1_{M^{j}} & 0 \\
0 & u_{\ell} \\
0 & v_{\ell} \\
0 & h_{j}
\end{array}\right)}{\longrightarrow} M^{j} \oplus M \oplus Z_{\ell} \oplus N_{j} \stackrel{\left(0, \nu_{j+1}, h_{j+1},-s_{j}\right)}{\longrightarrow} N_{j+1} \stackrel{\left(0, \sigma_{j}\right)}{\longrightarrow}\left(M^{j} \oplus Z_{\ell}\right)[1]
$$

is distinguished. For the series of morphisms $\omega_{j}: M^{j} \rightarrow N_{j}$ satisfying $\omega_{1}=\nu_{1}$ and $\omega_{j+1}=$ $\left(\nu_{j+1}, s_{j} \omega_{j}\right)$ for all $j$, this distinguished triangle is isomorphic to the triangle

$$
M^{j} \oplus Z_{\ell} \stackrel{\left(\begin{array}{cc}
1_{M^{j}} & 0 \\
0 & u_{\ell} \\
0 & v_{\ell} \\
\omega_{j} & h_{j}
\end{array}\right)}{\longrightarrow} M^{j} \oplus M \oplus Z_{\ell} \oplus N_{j} \stackrel{\left(\omega_{j+1}, \nu_{j+1}, h_{j+1},-s_{j}\right)}{\longrightarrow} N_{j+1} \stackrel{\left(0, \sigma_{j}\right)}{\longrightarrow}\left(M^{j} \oplus Z_{\ell}\right)[1]
$$

Indeed, we get morphisms of triangles

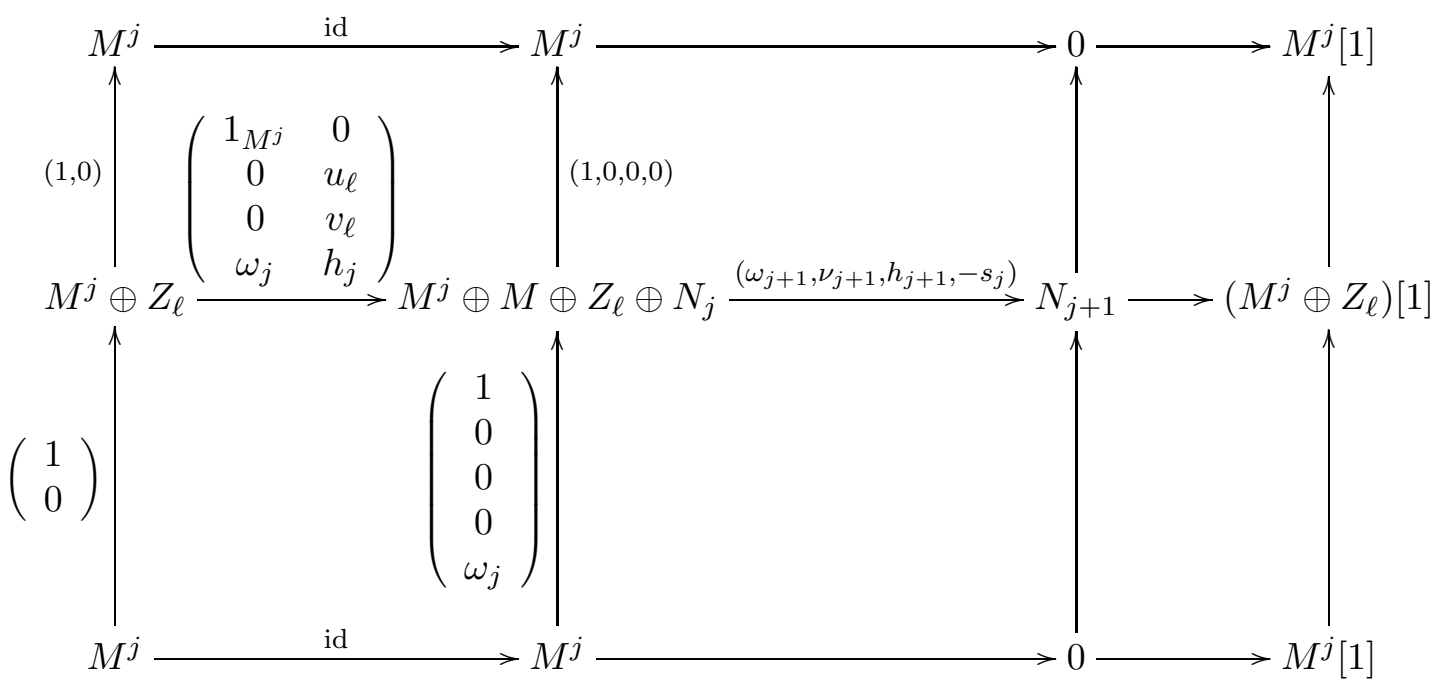

and therefore the middle triangle has a direct factor

$$
M^{j} \stackrel{\mathrm{id}}{\longrightarrow} M^{j} \longrightarrow 0 \longrightarrow M^{j}[1]
$$

and the remaining direct factor is the original distinguished triangle. 
Hence,

$$
\begin{gathered}
M^{j} \oplus Z_{\ell} \stackrel{\left(\begin{array}{cc}
1_{M^{j}} & 0 \\
0 & u_{\ell} \\
0 & v_{\ell}
\end{array}\right)}{\longrightarrow} M^{j+1} \oplus Z_{\ell} \\
\left.N_{j} \longrightarrow h_{j}\right) \downarrow_{\left(\omega_{j+1}, h_{j+1}\right)}^{\longrightarrow} N_{j+1}
\end{gathered}
$$

is a homotopy cartesian square. Moreover,

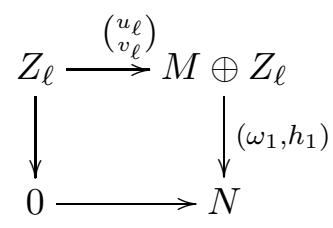

is a homotopy cartesian square by hypothesis. Now, since

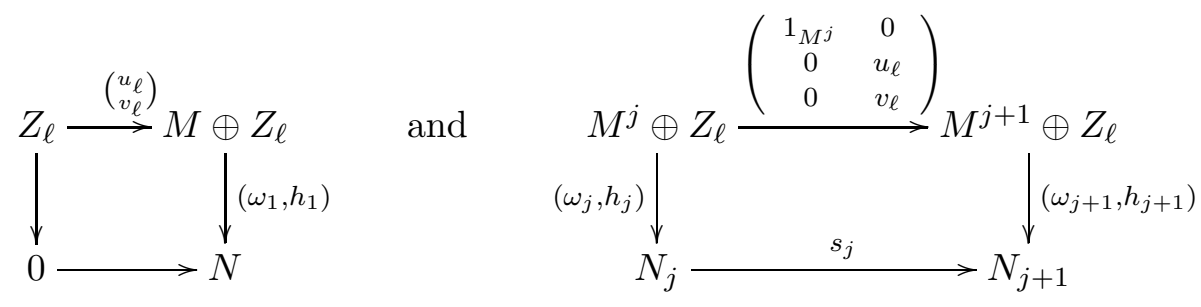

are homotopy cartesian squares, applying Lemma 8, we get a homotopy cartesian square

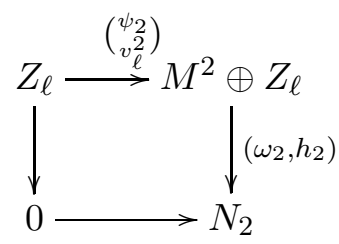

and by induction on $j$ we get that there is a morphism $\psi_{j}: Z_{\ell} \rightarrow M^{j}$ and a homotopy cartesian square

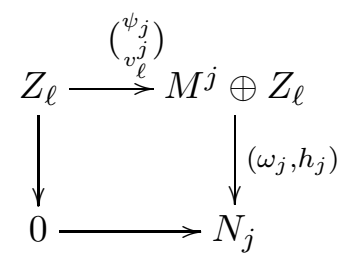

for all $j \in \mathbb{N}$. Hence for all $j>0$ there is a morphism $\psi_{j}: Z_{\ell} \rightarrow M^{j}$ such that

$$
Z_{\ell} \stackrel{\left(\begin{array}{c}
\psi_{j} \\
v_{\ell}^{j}
\end{array}\right)}{\longrightarrow} M^{j} \oplus Z_{\ell} \stackrel{\left(\omega_{j}, h_{j}\right)}{\longrightarrow} N_{j} \longrightarrow Z_{\ell}[1]
$$

is a distinguished triangle. Now $v_{\ell}$ is nilpotent of degree $k_{0}$, say. For any $k \geq k_{0}$ consider the commutative diagram with distinguished triangles in the horizontal rows

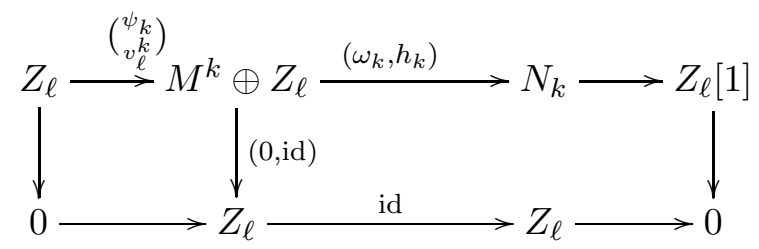


which can be completed by a map $z_{k}$ to a morphism of distinguished triangles, using TR3 (see e.g. [8, Definition 1.1.1] or [18, Definition 3.4.1]),

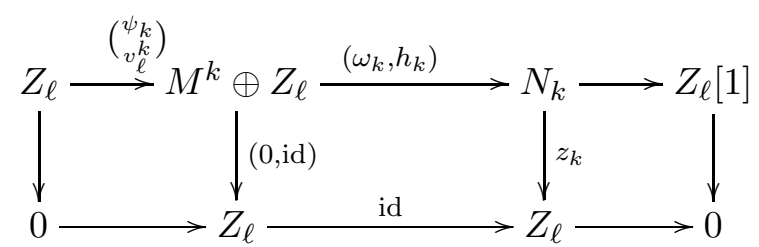

Therefore $z_{k} h_{k}=\mathrm{id}_{Z_{\ell}}$, and $h_{k}$ is a split monomorphism. We recall that we constructed the sequence $h_{j}$ as iterated homotopy pushouts, and hence, by definition we have a homotopy cartesian square

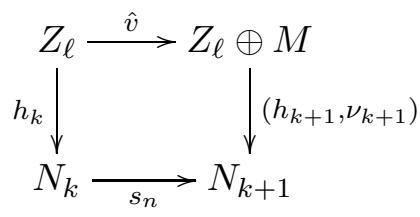

where $h_{k}$ (and $h_{k+1}$ ) are split monomorphisms. This shows first (cf e.g. [18, Lemma 3.4.9]) that $N_{k} \simeq Z_{\ell} \oplus C_{h_{k}}$, for $C_{h_{k}}$ being the mapping cone of $h_{k}$. Moreover, we get a distinguished triangle

$$
Z_{\ell} \stackrel{\left(\begin{array}{c}
\hat{v} \\
h_{k}
\end{array}\right)}{\longrightarrow} Z_{\ell} \oplus M \oplus N_{k} \stackrel{\left(h_{k+1}, \nu_{k+1},-s_{k}\right)}{\longrightarrow} N_{k+1} \longrightarrow Z_{\ell}[1]
$$

Since $h_{k}$ is split monomorphism, this distinguished triangle is isomorphic to the direct sum of the trivial distinguished triangle

$$
Z_{\ell} \stackrel{\mathrm{id}}{\longrightarrow} Z_{\ell} \longrightarrow 0 \longrightarrow Z_{\ell}[1]
$$

and

$$
0 \longrightarrow 0 \oplus C_{h_{k}} \oplus Z_{\ell} \oplus M \longrightarrow N_{k+1} \longrightarrow 0[1] .
$$

Hence (cf e.g. [18, Lemma 3.4.9]),

$$
\text { (†) } N_{k+1} \simeq C_{h_{k}} \oplus Z_{\ell} \oplus M \simeq N_{k} \oplus M
$$

Note that we could have argued also that since $z_{k}$ is left inverse to $h_{k},\left(0, z_{k}\right)$ is left inverse to $\left(\begin{array}{c}\hat{v} \\ h_{k}\end{array}\right)$, and so the above triangle splits. This then gives the desired isomorphism ( $\dagger$ ) via Remark 12,

Recall that, posing $N_{0}:=0$, for all $j \geq 1$ we have by construction homotopy cartesian squares

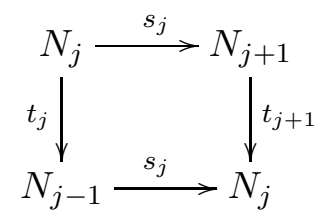

for all $j \geq 1$. Using Lemma 8 and an obvious induction as above this implies that we get an cartesian square

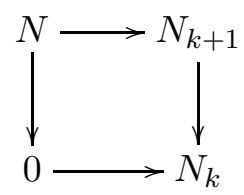

which gives a distinguished triangle

$$
N \longrightarrow N_{k+1} \longrightarrow N_{k} \longrightarrow N[1]
$$


where we have chosen $k$ such that $v_{\ell}^{k}=0$. By equation ( $\dagger$ ) we get $N_{k+1} \simeq N_{k} \oplus M$, which shows that there is a distinguished triangle

$$
N \longrightarrow N_{k} \oplus M \longrightarrow N_{k} \longrightarrow N[1]
$$

Posing $N_{k}=: Z_{r}$ this gives the statement, except that we do not get yet that the induced endomorphism of $Z_{r}$ is nilpotent.

Since the endomorphism ring of all objects in $\mathcal{T}$ are artinian and idempotents split, $\mathcal{T}$ is Krull-Schmidt (cf Proposition 10), and then we may split off $f^{\prime}$ in a nilpotent endomorphism of a direct factor and an automorphism of a direct factor, using Fitting's lemma. The automorphism part splits in the distinguished triangle, and we obtain the statement.

The other direction is done applying the statement proved above to the opposite category $\mathcal{T}^{o p}$ of $\mathcal{T}$.

Remark 13. A triangulated category with split idempotents for which each object has artinian endomorphism rings is Krull-Schmidt and a Fitting-like theorem holds (cf Remark 12). However, if $\mathcal{T}$ is a general triangulated category, and in particular if we do not assume that $\mathcal{T}$ is Krull-Schmidt, then we get a weaker statement in Theorem 11. The hypothesis that $\mathcal{T}$ has artinian endomorphism rings is only used at the very end of the proof of the theorem in order to be able to split off a direct factor in order to get a nilpotent endomorphism on the remaining factor. If $\mathcal{T}$ is a general triangulated category we proved that the existence of a distinguished triangle

$$
Z_{\ell} \stackrel{\left(\begin{array}{l}
u_{\ell} \\
v_{\ell}
\end{array}\right)}{\longrightarrow} M \oplus Z_{\ell} \longrightarrow N \longrightarrow Z_{\ell}[1]
$$

with nilpotent $v_{\ell}$ implies the existence of a distinguished triangle

$$
N \longrightarrow M \oplus Z_{r} \stackrel{\left(u_{r} v_{r}\right)}{\longrightarrow} Z_{r}[1] \longrightarrow N[1],
$$

but we are unable to deduce that $v_{r}$ is nilpotent.

\section{The Case of a CATEgory of COMPaCt objects in an algebraic COMPaCtly GENERATED CATEGORY}

Recall from Section 2.3 the construction of the category $\mathcal{A}[[T]]$. When we have a $\mathrm{dg}$ algebra with enough idempotents $A$ and view it as a dg category (see [10, Section 3]), we can undertake the same construction, but there is a classical notion of power series algebra $A[[T]]$ which does not correspond to the 'power series dg category' mentioned in Section 2.3 . The corresponding dg algebra with enough idempotents is the subalgebra of $A[[T]]$ given as

$$
\tilde{A}[[T]]=\bigoplus_{i, j \in I} \bigoplus_{n \in \mathbb{Z}} e_{i} A^{n} e_{j}[[T]] .
$$

That is, $\tilde{A}[[T]]$ consists of the power series $\sum_{k \in \mathbb{N}} a_{k} T^{k}$ for which there are finite subsets $J \subset I$ and $F \subset \mathbb{Z}$ such that $a_{k} \in \bigoplus_{i, j \in J} \bigoplus_{n \in F} e_{i} A^{n} e_{j}$, for all $k \in \mathbb{N}$. The subalgebra $\tilde{A}[[T]]$ is made into a dg algebra by defining $\tilde{A}[[T]]^{n}=\bigoplus_{i, j \in I} e_{i} A^{n} e_{j}[[T]]$, for each $n \in \mathbb{Z}$, and by defining its differential by the rule $d\left(\sum_{k \in \mathbb{N}} a_{k} T^{k}\right)=\sum_{i \in \mathbb{N}} d\left(a_{k}\right) T^{k}$. Note that then $\left(e_{i}\right)_{i \in I}$ is also a distinguished family of orthogonal idempotents of $\tilde{A}[[T]]$. Moreover, we have a canonical inclusion $\iota: A \hookrightarrow \tilde{A}[[T]]\left(a \rightsquigarrow a=a T^{o}\right)$ and a canonical augmentation map $\rho: \tilde{A}[[T]] \longrightarrow A\left(\sum_{k \in \mathbb{N}} a_{k} T^{k} \rightsquigarrow a_{0}\right)$ such that $\rho \circ \iota=1_{A}$. Both $\iota$ and $\rho$ are homomorphisms of dg algebras making the codomain into a unitary bimodule over the domain. We can apply to $\iota$ and $\rho$ the results of [10] (see Corollary 9.4 and Section 10 in that reference) concerning homomorphisms of dg algebras with enough idempotents. 
Likewise for right $A$-modules, there is a classical notion of 'module of power series' $M[[T]]$ which does not correspond the the ' $\mathrm{dg} \mathcal{A}[[T]]$-module of power series' mentioned in Section 2.3. The corresponding right $\operatorname{dg} \tilde{A}[[T]]$-module is

$$
\tilde{M}[[T]]=\bigoplus_{i \in I} \bigoplus_{n \in \mathbb{Z}} M^{n} e_{i}[[T]],
$$

which is the $K$-submodule of $M[[T]]$ consisting of those power series $\sum_{k \in \mathbb{N}} m_{k} T^{k}$ for which there exist finite subsets $J \subset I$ and $F \subset \mathbb{Z}$, both depending on the power series, such that $m_{k} \in \oplus_{i \in J, n \in F} M^{n} e_{i}$, for all $k \in \mathbb{N}$. Defining the grading by the rule $\tilde{M}[[T]]^{n}=$ $\bigoplus_{i \in I} M^{n} e_{i}[[T]]$ and the differential $d: \tilde{M}[[T]] \longrightarrow \tilde{M}[[T]]$ by the rule $d\left(\sum_{k \in \mathbb{N}} m_{k} T^{k}\right)=$ $\sum_{k \in \mathbb{N}} d\left(m_{k}\right) T^{k}$, we clearly endow $\tilde{M}[[T]]$ with a structure of right $\operatorname{dg} \tilde{A}[[T]]$-module. We warn the reader of the possible confusion of this construction when applied to the regular right $\operatorname{dg} A$-module $A_{A}$ for the resulting right $\operatorname{dg} \tilde{A}[[T]]$-module is not equal to the regular right dg module $\tilde{A}[[T]]_{\tilde{A}[[T]]}$.

4.1. Dualising degeneration data. We now give, and actually extend, the functor corresponding to ? $\hat{\otimes} V$ to the context of dg algebras with enough idempotents and $\mathrm{dg}$ modules over them.

Proposition 14. The assignment $M \rightsquigarrow \tilde{M}[[T]]$ is the definition on objects of a dg functor $? \hat{\otimes} V: D g-A \longrightarrow D g-\tilde{A}[[T]]$ which satisfies the following properties:

(1) ? $\hat{\otimes} V$ takes contractible $d g$ modules to contractible $d g$ modules.

(2) The associated functor on 0-cycle categories

$$
? \hat{\otimes} V: Z^{0}(D g-A)=\mathcal{C}(A) \longrightarrow \mathcal{C}(\tilde{A}[[T]])=Z^{0}(D g-\tilde{A}[[T]])
$$

is exact with respect to the respective abelian structures.

(3) ? $\hat{\otimes} V$ preserves acyclic $d g$ modules. In particular, it induces a triangulated functor $? \hat{\otimes} V: \mathcal{D}(A) \longrightarrow \mathcal{D}(\tilde{A}[[T]])$ which is both the left and right derived functor of 'itself'. We will denote this functor by $\uparrow_{K}^{V}$.

(4) The multiplication map $M \otimes_{A} \tilde{A}[[T]] \longrightarrow \tilde{M}[[T]]$ defines a homological natural transformation of $d g$ functors $\mu: \iota^{*} \longrightarrow ? \hat{\otimes} V$

(5) The induced natural transformation $\mu: \mathbb{L} \iota^{*} \longrightarrow \uparrow_{K}^{V}$ of triangulated functors $\mathcal{D}(A) \longrightarrow$ $\mathcal{D}(\tilde{A}[[T]])$ is a natural isomorphism when evaluated at objects of $\operatorname{per}(A)$.

Proof. If $f: M \longrightarrow N$ is a homogeneous morphism in $D g-A$, we define $\tilde{f}:=(? \hat{\otimes} V)(f)$ by the rule $\tilde{f}\left(\sum_{k \in \mathbb{N}} m_{k} T^{k}\right)=\sum_{k \in \mathbb{N}} f\left(m_{k}\right) T^{k}$. It is routine to verify that the assignments $M \rightsquigarrow \tilde{M}[[T]]$ and $f \rightsquigarrow \tilde{f}$ give a graded functor $G R-A \longrightarrow G R-\tilde{A}[[T]]$. In order to check that they define a dg functor ? $\hat{\otimes} V: D g-A \longrightarrow D g-\tilde{A}[[T]]$, we need to check that if $M, N$ are right $\operatorname{dg} A$-modules and $d: \operatorname{HOM}_{A}(M, N) \longrightarrow \operatorname{HOM}_{A}(M, N)$ and $\delta$ : $\operatorname{HOM}_{\tilde{A}[[T]]}(\tilde{M}[[T]], \tilde{N}[[T]]) \longrightarrow \operatorname{HOM}_{\tilde{A}[[T]]}(\tilde{M}[[T]], \tilde{N}[[T]])$ are the respective differentials on Hom spaces, then one has $\delta(\tilde{f})=\widetilde{d(f)}$, for any homogeneous element $f \in \operatorname{HOM}_{A}(M, N)$. On one hand, we have that

$$
\delta(\tilde{f})=d_{\tilde{N}[[T]]} \circ \tilde{f}-(-1)^{|f|} \tilde{f} \circ d_{\tilde{M}[[T]]} .
$$

On the other hand, if we let act $\widetilde{d(f)}$ on a homogeneous element $\sum_{k \in \mathbb{N}} m_{k} T^{k} \in \tilde{M}[[T]]$ (whence the degree $\operatorname{deg}\left(m_{k}\right)$ is independent of $k$ ), then we get: 


$$
\begin{aligned}
\widetilde{d(f)}\left(\sum_{k \in \mathbb{N}} m_{k} T^{k}\right) & =\sum_{k \in \mathbb{N}} d(f)\left(m_{k}\right) T^{k} \\
& =\sum_{k \in \mathbb{N}}\left[\left(d_{N} \circ f-(-1)^{|f|} f \circ d_{M}\right)\left(m_{k}\right)\right] T^{k} \\
& =\sum_{k \in \mathbb{N}} d_{N}\left(f\left(m_{k}\right)\right) T^{k}-(-1)^{|f|} \sum_{k \in \mathbb{N}} f\left(d_{M}\left(m_{k}\right)\right) T^{k} \\
& =d_{\tilde{N}[[T]]}\left(\sum_{k \in \mathbb{N}} f\left(m_{k}\right) T^{k}\right)-(-1)^{|f|} \tilde{f}\left(\sum_{k \in \mathbb{N}} d_{M}\left(m_{k}\right) T^{k}\right) \\
& =\left[d_{\tilde{N}[[T]]} \circ \tilde{f}-(-1)^{|f|} \tilde{f} \circ d_{\tilde{M}[[T]]}\right]\left(\sum_{k \in \mathbb{N}} m_{k} T^{k}\right) .
\end{aligned}
$$

This shows that $\delta(\tilde{f})=\widetilde{d(f)}$, as desired.

Finally, it is also routine to see that $(? \hat{\otimes} V)\left(\operatorname{cone}\left(1_{M}\right)\right) \cong \operatorname{cone}\left(1_{(? \hat{\otimes} V)(M)}\right)$, which ends the proof of assertion (1).

(2) Let $L \stackrel{f}{\longrightarrow} M \stackrel{g}{\longrightarrow} N$ be an exact sequence in $\mathcal{C}(A)$, when this category is considered with its natural abelian structure, and consider the corresponding sequence $\tilde{L}[[T]] \stackrel{\tilde{f}}{\longrightarrow}$ $\tilde{M}[[T]] \stackrel{\tilde{g}}{\longrightarrow} \tilde{N}[[T]]$. Since $\tilde{f}$ and $\tilde{g}$ are both morphisms in $G r-\tilde{A}[[T]]$ we just need to check that the induced sequence

$$
\tilde{L}[[T]]^{n} e_{i}=L^{n} e_{i}[[T]] \stackrel{\tilde{f}}{\longrightarrow} \tilde{M}[[T]]^{n} e_{i}=M^{n} e_{i}[[T]] \stackrel{\tilde{g}}{\longrightarrow} \tilde{N}[[T]]^{n} e_{i}=N^{n} e_{i}[[T]]
$$

is exact, for all $i \in I$ and $n \in \mathbb{Z}$. But, given $\sum_{k \in \mathbb{N}} m_{k} T^{k} \in M^{n} e_{i}[[T]]$, we have that $\tilde{g}\left(\sum_{k \in \mathbb{N}} m_{k} T^{k}\right)=0$ if and only if $g\left(m_{k}\right)=0$ for all $k \in \mathbb{N}$. This in turn is equivalent to saying that, for each $k \in \mathbb{N}$, there exists a $l_{k} \in L^{n} e_{i}$ such that $f\left(l_{k}\right)=m_{k}$. That is, we have that $\sum_{k \in \mathbb{N}} m_{k} T^{k} \in \operatorname{Ker}(\tilde{g})$ if and only if $\sum_{k \in \mathbb{N}} m_{k} T^{k}=\tilde{f}\left(\sum_{k \in \mathbb{N}} l_{k} T^{k}\right)$, for some $\sum_{k \in \mathbb{N}} l_{k} T^{k} \in L^{n} e_{i}[[T]]$.

(3) Let $M$ be an acyclic right dg $A$-module, let $\sum_{k \in \mathbb{N}} m_{k} T^{k}$ be an element of $\operatorname{Ker}\left(d^{n}\right.$ : $\tilde{M}[[T]]^{n} \longrightarrow \tilde{M}[[T]]^{n+1}$ ) and let $J \subset I$ be a finite subset such that $m_{k} \in \bigoplus_{i \in J} M^{n} e_{i}$, for all $k \in \mathbb{N}$. By the acyclicity condition of $M$, for each $k \in \mathbb{N}$, we have an $m_{k}^{\prime} \in$ $\bigoplus_{i \in J} M^{n-1} e_{i}$ such that $d\left(m_{k}^{\prime}\right)=m_{k}$. It follows $\sum_{k \in \mathbb{N}} m_{k}^{\prime} T^{k}$ is an element of $\tilde{M}[[T]]^{n}$ such that $d\left(\sum_{k \in \mathbb{N}} m_{k}^{\prime} T^{k}\right)=\sum_{k \in \mathbb{N}} m_{k} T^{k}$. Therefore $\tilde{M}[[T]]$ is an acyclic right $\operatorname{dg} \tilde{A}[[T]]$-module. The last comment of the assertion follows from [10, Remark 7.11].

(44) We clearly have that $\mu_{M}: M \otimes_{A} \tilde{A}[[T]] \longrightarrow \tilde{M}[[T]]$ is a morphism (of zero degree) in $G R-\tilde{A}[[T]]$. In addition, if we denote by $d: M \otimes_{A} \tilde{A}[[T]] \longrightarrow M \otimes_{A} \tilde{A}[[T]]$ and $\tilde{d}: \tilde{M}[[T]] \longrightarrow \tilde{M}[[T]]$ the respective differentials, then, for all homogeneous elements $m \in M$ 
and $\sum_{k \in \mathbb{N}} a_{k} T^{k}$, we have

$$
\begin{aligned}
\left(\mu_{M} \circ d\right)\left[m \otimes\left(\sum_{k \in \mathbb{N}} a_{k} T^{k}\right)\right] & =\mu_{M}\left(d_{M}(m) \otimes\left(\sum_{k \in \mathbb{N}} a_{k} T^{k}\right)+(-1)^{|m|} m \otimes\left(\sum_{k \in \mathbb{N}} d\left(a_{k}\right) T^{k}\right)\right) \\
& =\sum_{k \in \mathbb{N}} d_{M}(m) a_{k} T^{k}+(-1)^{|m|} m d\left(a_{k}\right) T^{k} \\
& =\sum_{k \in \mathbb{N}}\left(d_{M}(m) a_{k}+(-1)^{|m|} m d\left(a_{k}\right)\right) T^{k} \\
& =\sum_{k \in \mathbb{N}} d_{M}\left(m a_{k}\right) T^{k} \\
& =\tilde{d}\left(\sum_{k \in \mathbb{N}} m a_{k} T^{k}\right) \\
& =\left(\tilde{d} \circ \mu_{M}\right)\left[m \otimes\left(\sum_{k \in \mathbb{N}} a_{k} T^{k}\right)\right] .
\end{aligned}
$$

Then, once the naturality $\mu$ is proved, we will have that it is actually a homological natural transformation of dg functors (see [10, Remark 7.1]). But that naturality is clear since we have

$$
\begin{aligned}
\left(\tilde{f} \circ \mu_{M}\right)\left[m \otimes\left(\sum_{k \in \mathbb{N}} a_{k} T^{k}\right)\right] & =\tilde{f}\left(\sum_{k \in \mathbb{N}} m a_{k} T^{k}\right)=\sum_{k \in \mathbb{N}} f\left(m a_{k}\right) T^{k}=\sum_{k \in \mathbb{N}} f(m) a_{k} T^{k} \\
& =\mu_{N}\left[f(m) \otimes\left(\sum_{k \in \mathbb{N}} a_{k} T^{k}\right)\right] \\
& =\left(\mu_{N} \circ\left(f \otimes 1_{\tilde{A}[[T]]}\right)\right)\left[m \otimes\left(\sum_{k \in \mathbb{N}} a_{k} T^{k}\right)\right],
\end{aligned}
$$

for any homogeneous morphism $f: M \longrightarrow N$ in $G R-A$ and all homogeneous elements $m \in M$ and $\sum_{k \in \mathbb{N}} a_{k} T^{k} \in \tilde{A}[[T]]$.

(5) Let $\Pi_{A}: \mathcal{D}(A) \longrightarrow \mathcal{H}(A)$ denote the homotopically projective resolution functor. Since each $e_{i} A$ is homotopically projective (see [10, Example 7.6]), we have that $\Pi_{A}\left(e_{i} A\right) \cong$ $e_{i} A$ in $\mathcal{H}(A)$. Moreover, we have an isomorphism $\mu_{e_{i} A}: e_{i} A \otimes_{A} \tilde{A}[[T]] \stackrel{\cong}{\longrightarrow} e_{i} \tilde{A}[[T]]=e_{i} A \hat{\otimes} V$ in $D g-\tilde{A}[[T]]$ (see the proof of Proposition 10.5 in [10]). One then gets from [10, Proposition 7.12] that $\mu_{e_{i} A}: \mathbb{L} \iota^{*}\left(e_{i} A\right) \longrightarrow\left(e_{i} A\right) \uparrow_{K}^{V}$ is an isomorphism in $\mathcal{D}(\tilde{A}[[T]])$, from which it follows that $\mu_{M}: \mathbb{L}_{\iota}{ }^{*}(M) \longrightarrow M \uparrow_{K}^{V}$ is an isomorphism, for all $M \in \operatorname{per}(A)=\operatorname{thick}_{\mathcal{D}(A)}\left(e_{i} A: i \in\right.$ $I)$.

Note that we have a canonical isomorphism of dg algebras with enough idempotents

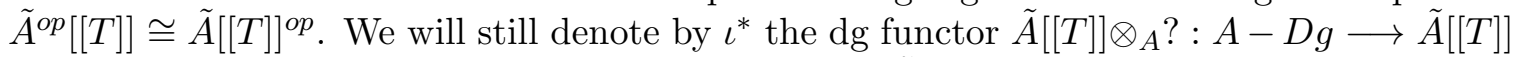
and by $\mathbb{L} \iota^{*}$ its left derived functor $\mathcal{D}\left(A^{o p}\right) \longrightarrow \mathcal{D}\left(\tilde{A}[[T]]^{o p}\right)$. We will denote by $V \hat{\otimes}$ ? : $A-D g \longrightarrow \tilde{A}[[T]]-D g$ and $\uparrow_{k}^{V}: \mathcal{D}\left(A^{o p}\right) \longrightarrow \mathcal{D}\left(\tilde{A}[[T]]^{o p}\right)$ the corresponding dg functor and triangulated functor, respectively. We now get:

Corollary 15. Consider the compositions of triangulated functors

$$
\mathcal{D}(A)^{o p} \stackrel{\mathbb{R} H o m_{A}(?, A)}{\longrightarrow} \mathcal{D}\left(A^{o p}\right) \stackrel{\mathbb{L} \iota^{*}}{\longrightarrow} \mathcal{D}\left(\tilde{A}[[T]]^{o p}\right)
$$

and

$$
\left.\mathcal{D}(A)^{o p} \stackrel{\left(\mathbb{L} \iota^{*}\right)^{o}}{\longrightarrow} \mathcal{D}(\tilde{A}[[T]])^{o p} \stackrel{\mathbb{R} H o m_{\tilde{A}[[T]]}}{\longrightarrow} \stackrel{?}{\longrightarrow} \tilde{A}[[T]]\right)^{D}\left(\tilde{A}[[T]]^{o p}\right)
$$


There are natural isomorphisms of triangulated functors

and

$$
\eta:\left(\mathbb{L} \iota_{\iota}^{*} \circ \mathbb{R} \operatorname{Hom}_{A}(?, A)\right)_{\mid p e r(A)^{o p}} \stackrel{\cong}{\longrightarrow}\left[\mathbb{R} \operatorname{Hom}_{\tilde{A}[[T]]}(?, \tilde{A}[[T]]) \circ \mathbb{L}^{*}\right]_{\mid p e r(A)^{o p}}
$$

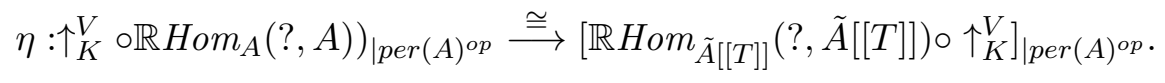

Proof. The first natural isomorphism is a direct consequence of [10, Proposition 10.5]. On the other hand, Proposition 14 and its left-right symmetric version gives natural isomorphisms $\left(\mathbb{L} \iota^{*}\right)_{\mid \operatorname{per}(A)} \cong\left(\uparrow_{K}^{V}\right)_{\mid \operatorname{per}(A)}$ and $\left(\mathbb{L} \iota^{*}\right)_{\mid \operatorname{per}\left(A^{o p}\right)} \cong\left(\uparrow_{K}^{V}\right)_{\mid \operatorname{per}\left(A^{o p}\right)}$. Using now the duality

$$
\mathbb{R H o m}_{A}(?, A): \operatorname{per}(A) \stackrel{\cong o}{\longrightarrow} \operatorname{per}\left(A^{o p}\right),
$$

the result follows.

We now revisit and generalize some point of [11. Note that the variable $T$ is not an element of $\tilde{A}[[T]]$, unless $A$ has a unit. However, if $Q$ is a right $\operatorname{dg} \tilde{A}[[T]]$-module and $x \in Q$ is a homogeneous element, then the product $x T$ makes sense. Indeed since $x=\sum_{i \in I} x e_{i}$, with $x e_{i}=0$ for almost all $i \in I$, the element $x T:=\sum_{i \in I} x\left(e_{i} T\right)$ is a well-defined element of $Q$ with $\operatorname{deg}(x T)=\operatorname{deg}(x)$. Furthermore, if $f: Q \longrightarrow Q^{\prime}$ is a morphism in $D g-\tilde{A}[[T]]$, then we have $f(x T)=f\left(\sum_{i \in I} x\left(e_{i} T\right)\right)=\sum_{i \in I} f(x) e_{i} T=f(x) T$. We can now prove:

Lemma 16. For each right $d g \tilde{A}[[T]]$-module $Q$, the map $t_{Q}: Q \longrightarrow Q(x \rightsquigarrow x T)$ is a morphism of zero degree in $D g-\tilde{A}[[T]]$ and, when $Q$ varies, the $t_{Q}$ give a homological natural transformation of dg functors $t: 1_{D g-\tilde{A}[[T]]} \longrightarrow 1_{D g-\tilde{A}[[T]]}$.

Proof. Let $x \in Q$ and $\sum_{k \in \mathbb{N}} a_{k} T^{k} \in \tilde{A}[[T]]$ be homogeneous elements. By definition of $\tilde{A}[[T]]$ and by the fact that $Q=\bigoplus_{i \in I} Q e_{i}$, we have a finite subset $F \subset I$ such that $x e_{i}=0$ and $a_{k} e_{i}=0$, for all $i \in I \backslash F$ and all $k \in \mathbb{N}$. It follows that $\left(x \sum_{k \in \mathbb{N}} a_{k} T^{k}\right) e_{i}=0$, for all $i \in I \backslash F$. We then have

$$
\begin{aligned}
t_{Q}\left(x \sum_{k \in \mathbb{N}} a_{k} T^{k}\right) & =\left(x \sum_{k \in \mathbb{N}} a_{k} T^{k}\right) T=\sum_{i \in F}\left(x \sum_{k \in \mathbb{N}} a_{k} T^{k}\right) e_{i} T=\sum_{i \in F} x\left(\sum_{k \in \mathbb{N}} a_{k} e_{i} T^{k+1}\right) \\
& =x \sum_{k \in \mathbb{N}} a_{k} T^{k+1}=(x T) \sum_{k \in \mathbb{N}} a_{k} T^{k}=t_{Q}(x) \sum_{k \in \mathbb{N}} a_{k} T^{k},
\end{aligned}
$$

which shows that $t_{Q}$ is a morphism of zero degree in $D g-\tilde{A}[[T]]$.

If $f: Q \longrightarrow Q^{\prime}$ is a homogeneous morphism in $D g-\tilde{A}[[T]]$ then we have $\left(t_{Q^{\prime}} \circ f\right)(x)=$ $f(x) T=f(x T)=\left(f \circ t_{Q}\right)(x)$, for each $x \in Q$. This proves that, when $Q$ varies, the $t_{Q}$ give a natural transformation of $\mathrm{dg}$ functors $t: 1_{D g-\tilde{A}[[T]]} \longrightarrow 1_{D g-\tilde{A}[[T]]}$. This natural transformation is homological since we have

$$
\begin{aligned}
\left(d_{Q} \circ t_{Q}\right)(x) & =d_{Q}(x T)=d_{Q}\left(\sum_{i \in I} x\left(e_{i} T\right)\right)=\sum_{i \in I} d_{Q}\left(x\left(e_{i} T\right)\right) \\
& =\sum_{i \in I}\left(d_{Q}(x) e_{i}\right) T=d_{Q}(x) T=\left(t_{Q} \circ d_{Q}\right)(x),
\end{aligned}
$$

for each homogeneous element $x \in Q$, due to the fact that $d_{\tilde{A}[[T]]}\left(e_{i} T\right)=d\left(e_{i}\right) T=0$ (see [10, Remark 7.1]).

Note that the associated natural transformation of triangulated functors $t: 1_{\mathcal{D}(\tilde{A}[[T]])} \longrightarrow$ $1_{\mathcal{D}(\tilde{A}[[T]])}$ is the one given in [1], after translation to the language of dg algebras with enough idempotents. Replacing $A$ by $A^{o p}$ in Lemma [16 and interpreting right $\mathrm{dg}$ modules over $\tilde{A}[[T]]^{o p} \cong \tilde{A}^{o p}[[T]]$ as left $\operatorname{dg} \tilde{A}[[T]]$-modules, we get a natural transformation of $\mathrm{dg}$ functors $t: 1_{\tilde{A}[[T]]-D g} \longrightarrow 1_{\tilde{A}[[T]]-D g}$ which in turn gives a natural transformation of triangulated functors $t: 1_{\mathcal{D}\left(\tilde{A}[[T]]^{o p}\right)}^{\longrightarrow} 1_{\mathcal{D}\left(\tilde{A}[[T]]^{o p}\right)}$. These natural transformations do not have 
correspondents for $\operatorname{dg} \tilde{A}[[T]]-\tilde{A}[[T]]$-bimodules, because the action of $T$ by multiplication on a $\operatorname{dg} \tilde{A}[[T]]-\tilde{A}[[T]]$-bimodule need not be the same on the left and on the right. We say that a dg $\tilde{A}[[T]]-\tilde{A}[[T]]$-bimodule $X$ is $T$-symmetric when $T x=x T$, for each $x \in X$. Note that, with a suitable modification of the argument used in the proof of Lemma 16, one easily sees that if $X$ is a $T$-symmetric $\operatorname{dg} \tilde{A}[[T]]$-bimodule, then the assignment $x \rightsquigarrow x T=T x$ is a morphism of $\tilde{A}[[T]]-\tilde{A}[[T]]$-bimodules, which we also denote by $t_{X}$.

Proposition 17. Let us consider the bi-triangulated functor

$$
\mathbb{R} H O M_{\tilde{A}[[T]]}(?, ?): \mathcal{D}(\tilde{A}[[T]])^{o p} \otimes \mathcal{D}\left(\tilde{A}[[T]] \otimes \tilde{A}[[T]]^{o p}\right) \longrightarrow \mathcal{D}\left(\tilde{A}[[T]]^{o p}\right)
$$

(see Proposition Q Q) and let $Q$ be a right $d g \tilde{A}[[T]]$-module and $X$ be a T-symmetric $d g$ $\tilde{A}[[T]]-\tilde{A}[[T]]$-bimodule.

Then $\mathbb{R} H O M_{\tilde{A}[[T]]}\left(t_{Q}^{o}, 1_{X}\right)$ and $\mathbb{R} H O M_{\tilde{A}[[T]]}\left(1_{Q}^{o}, t_{X}\right)$, considered as maps

$$
\mathbb{R} H O M_{\tilde{A}[[T]]}(Q, X) \longrightarrow \mathbb{R} H O M_{\tilde{A}[[T]]}(Q, X)
$$

are equal. Moreover they are equal to the evaluation of the natural transformation

$$
t: 1_{\mathcal{D}\left(\tilde{A}[[T]]^{o p}\right)} \longrightarrow 1_{\mathcal{D}\left(\tilde{A}[[T]]^{o p}\right)}
$$

at $\mathbb{R} H O M_{\tilde{A}[[T]]}(Q, X)$.

Proof. By Proposition 2, we have a natural isomorphism of triangulated functor

$$
\mathbb{R H O M}_{\tilde{A}[[T]]}(?, X) \cong \mathbb{R H o m}_{\tilde{A}[[T]]}(?, X) .
$$

So in order to see that $\mathbb{R H O M}_{\tilde{A}[[T]]}\left(t_{Q}^{o}, 1_{X}\right)$ is the evaluation of $t$ at $\mathbb{R H O M}_{\tilde{A}[[T]]}(Q, X)$ it is enough to check that $t_{Q}^{*}=\mathbb{R H o m}_{\tilde{A}[[T]]}(?, X)\left(t_{Q}\right)$ is precisely $t_{\mathbb{R} \operatorname{Hom}_{\tilde{A}[T]]}(Q, X)}$, where $\mathbb{R H o m}_{\tilde{A}[[T]]}(Q, X):=\mathbb{R H o m}_{\tilde{A}[[T]]}(?, X)(Q)$ in the rest of the proof. Note that $t_{Q}^{*}$ is the map $\mathbb{R H o m}_{\tilde{A}[[T]]}(Q, X)=\overline{H O M}_{\tilde{A}[[T]]}(\Pi(Q), X) \stackrel{\Pi\left(t_{Q}\right)^{*}}{\longrightarrow} \overline{H O M}_{\tilde{A}[[T]]}(\Pi(Q), X)=\mathbb{R H o m}_{\tilde{A}[[T]]}(Q, X)$. Here and in the rest of the proof

$$
\Pi:=\Pi_{\tilde{A}[[T]]}: \mathcal{D}(\tilde{A}[[T]]) \longrightarrow \mathcal{H}(\tilde{A}[[T]])
$$

and

$$
\Upsilon:=\Upsilon_{\tilde{A}[[T]] \otimes \tilde{A}[[T]]^{o p}}: \mathcal{D}\left(\tilde{A}[[T]] \otimes \tilde{A}[[T]]^{o p}\right) \longrightarrow \mathcal{H}\left(\tilde{A}[[T]] \otimes \tilde{A}[[T]]^{o p}\right)
$$

are the homotopically projective and the homotopically injective resolution functors, respectively.

It is convenient to have a careful look at a special case of the action of $\Pi$ and $\Upsilon$ on morphisms. Let $Q$ and $X$ be as in the statement and let $f: Q \longrightarrow Q$ and $\alpha: X \longrightarrow X$ be morphisms in $\mathcal{H}(\tilde{A}[[T]])$ and $\mathcal{H}\left(\tilde{A}[[T]] \otimes \tilde{A}[[T]]^{o p}\right)$, respectively. Abusing notation, we put $q(f)=f$ and $q(\alpha)=\alpha$, where $q$ is the functor from the homotopy to the derived category in each case. Viewing $Q$ and $X$ as objects of the respective derived categories, we have a counit map

and a unit map

$$
\pi_{Q}:(\Pi \circ q)(Q)=\Pi(Q) \longrightarrow Q
$$

$$
\iota_{X}: X \longrightarrow(\Upsilon \circ q)(X)=\Upsilon(X),
$$

which are quasi-isomorphism. Then $\Pi(f):=(\Pi \circ q)(f)$ is a morphism $\Pi(Q) \longrightarrow \Pi(Q)$ in $\mathcal{H}(\tilde{A}[[T]])$ such that

$$
\pi_{Q} \circ \Pi(f)=f \circ \pi_{Q}(*),
$$

due to the naturality of the counit $\pi$. But since we have an isomorphism

$$
\operatorname{Hom}_{\mathcal{H}(\tilde{A}[[T]])}(\Pi(Q), \Pi(Q)) \stackrel{\cong}{\longrightarrow} \operatorname{Hom}_{\mathcal{D}(\tilde{A}[[T]])}(Q, Q)
$$


(which maps $\varphi \rightsquigarrow q(\pi) \circ \varphi \circ q(\pi)^{-1}$ ), we see that $\Pi(f)$ is the unique morphism in $\mathcal{H}(\tilde{A}[[T]])$ satisfying the equality $\left({ }^{*}\right)$. Similarly, $\Upsilon(\alpha)$ is the unique morphism $\Upsilon(X) \longrightarrow \Upsilon(X)$ in $\left.\mathcal{H}(\tilde{A}[[T]]) \otimes \tilde{A}[[T]]^{o p}\right)$ such that

$$
\Upsilon(\alpha) \circ \iota_{X}=\iota_{X} \circ \alpha
$$

By taking $f=t_{Q}$ in this argument, we readily see that $\Pi\left(t_{Q}\right)=t_{\Pi(Q)}$ since, due to the naturality of $t: 1_{D g-\tilde{A}[[T]]} \longrightarrow 1_{D g-\tilde{A}[[T]]}$, we have that $\pi \circ t_{\Pi(Q)}=t_{Q} \circ \pi$ in $\mathcal{H}(\tilde{A}[[T]])$. The analogous fact does not work for $\alpha=t_{X}$ since we do not have a correspondent of the natural transformation $t$ for $\tilde{A}[[T]]-\tilde{A}[[T]]$-bimodules. In any case, these comments together with the previous paragraph show that $\mathbb{R H o m}_{\tilde{A}[[T]]}(?, X)\left(t_{Q}\right)=t_{\Pi(Q)}^{*}$. Using the naturality of $t$, we see that $t_{\Pi(Q)}^{*}$ is a morphism $\overline{\mathrm{HOM}}_{A}(\Pi(Q), X) \longrightarrow \overline{\mathrm{HOM}}_{A}(\Pi(Q), X)$ of left $\operatorname{dg} \tilde{A}[[T]]$-modules which maps

$$
f \rightsquigarrow(-1)^{\left|t_{\Pi(Q)}\right||f|} f \circ t_{\Pi(Q)}=f \circ t_{\Pi(Q)}=t_{X} \circ f .
$$

We then have that

$$
\left[t_{\Pi(Q)}^{*}(f)\right](z)=\left(f \circ t_{X}\right)(z)=f(z T)=f(z) T=T f(z)=(T f)(z)=\left[t_{\overline{\operatorname{HOM}}_{A}(\Pi(Q), X)}(f)\right](z),
$$

for all homogeneous elements $z \in \Pi(Q)$, using the definition of the left $\tilde{A}[[T]]$-module structure on $\overline{\mathrm{HOM}}_{A}(\Pi(Q), X)$ (see [10, Section 8]) and the $T$-symmetry of $X$. Therefore we have $\mathbb{R H o m}_{A}(?, X)\left(t_{Q}\right)=t_{\mathbb{R H o m}}(Q, X)$, as desired.

On the other hand, by Proposition 2, there is a natural isomorphism

$$
\mathbb{R H O M}_{\tilde{A}[[T]]}(Q, ?) \cong \mathbb{R H o m}_{\tilde{A}[[T]]}(\Pi(Q), ?)=q \circ \overline{H O M}_{\tilde{A}[[T]]}(\Pi(Q), ?) \circ \Upsilon
$$

of triangulated functors

$$
\mathcal{D}\left(\tilde{A}[[T]] \otimes \tilde{A}[[T]]^{o p}\right) \longrightarrow \mathcal{D}\left(\tilde{A}[[T]]^{o p}\right) .
$$

Then $\mathbb{R H O M}_{\tilde{A}[[T]]}\left(1_{Q}^{o}, t_{X}\right)$ is the morphism

$$
\begin{aligned}
\overline{H O M}_{\tilde{A}[[X]]}(\Pi(Q), \Upsilon(X)) & \longrightarrow \overline{H O M}_{\tilde{A}[[X]]}(\Pi(Q), \Upsilon(X)) \\
f & \left.\rightsquigarrow(-1)^{\left|1_{Q}\right| \mid q\left(\Upsilon\left(t_{X}\right) \mid\right.} \Upsilon\left(t_{X}\right) \circ f \circ 1_{Q}\right)=\Upsilon\left(t_{X}\right)_{*}(f) .
\end{aligned}
$$

In other words, we have that

$$
\mathbb{R H O M}_{\tilde{A}[[T]]}\left(1_{Q}^{o}, t_{X}\right)=q\left(\Upsilon\left(t_{X}\right)_{*}\right),
$$

where $q: \mathcal{H}\left(\tilde{A}[[T]]^{o p}\right) \longrightarrow \mathcal{D}\left(\tilde{A}[[T]]^{o p}\right)$ is the canonical functor and

$$
\Upsilon\left(t_{X}\right)_{*}=\overline{H O M}_{A}(\Pi(M), ?)\left(\Upsilon\left(t_{X}\right)\right): \overline{H O M}_{A}(\Pi(M), \Upsilon(X)) \longrightarrow \overline{H O M}_{A}(\Pi(M), \Upsilon(X)) \text {. }
$$

But the induced functor

$$
\overline{H O M}_{\tilde{A}[[T]]}(\Pi(M), ?): \mathcal{H}\left(\tilde{A}[[T]] \otimes \tilde{A}[[T]]^{o p}\right) \longrightarrow \mathcal{H}\left(\tilde{A}[[T]]^{o p}\right)
$$

preserves quasi-isomorphisms since $\Pi(M)$ is homotopically projective in $\mathcal{H}(\tilde{A}[[T]])$. If now $\iota:=\iota_{X}: X \longrightarrow \Upsilon(X)$ is as above, then

$$
\left.\iota_{*}:=\overline{H O M}_{\tilde{A}[[T]]}(\Pi(M), \iota): \overline{H O M}_{\tilde{A}[[X]]}(\Pi(Q), X) \longrightarrow \overline{H O M}_{\tilde{A}[[X]]}(\Pi(Q), \Upsilon(X))\right)
$$

is a quasi-isomorphism of left $\operatorname{dg} \tilde{A}[[T]]$-modules. Applying to the equality $\Upsilon\left(t_{X}\right) \circ \iota=\iota \circ t_{X}$ the functor

$$
\overline{H O M}_{A}(\Pi(M), ?): \mathcal{H}\left(\tilde{A}[[T]] \otimes \tilde{A}[[T]]^{o p}\right) \longrightarrow \mathcal{H}\left(\tilde{A}[[T]]^{o p}\right)
$$


we get the following commutative diagram in $\mathcal{H}\left(\tilde{A}[[T]]^{o p}\right)$, where the horizontal arrows are quasi-isomorphisms.

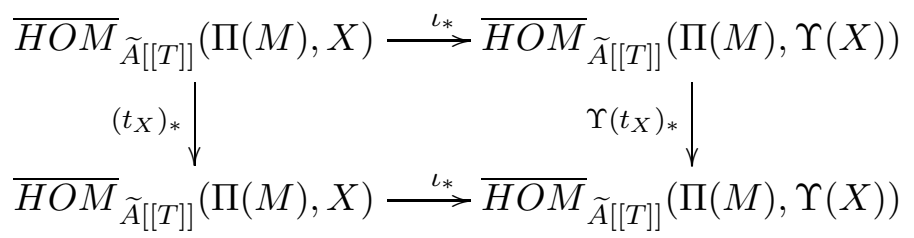

Moreover, the left vertical arrow takes $f \rightsquigarrow t_{X} \circ f$, for each homogeneous element $f \in$ $\overline{H O M}_{\tilde{A}[[T]]}(\Pi(M), X)$. But, in turn, we have that

$$
\left(t_{X} \circ f\right)(v)=T f(v)=(T f)(v)=t_{\overline{H O M}_{\tilde{A}[T]]}(\Pi(M), X)}(f)(v),
$$

for each homogeneous element $v \in \Pi(M)$. Therefore the left vertical arrow of last diagram is the evaluation of the natural transformation of dg functors $t: 1_{\tilde{A}[[T]]-D g} \longrightarrow 1_{\tilde{A}[[T]]-D g}$ at $\overline{H O M}_{\tilde{A}[[T]]}(\Pi(Q), X)$. The fact that $t$ is a natural transformation of $\mathrm{dg}$ functors implies that we also have an equality

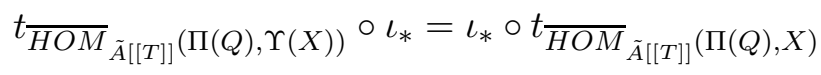

in $\tilde{A}[[T]]-D g$ and, hence, also in $\mathcal{H}\left(\tilde{A}[[T]]^{o p}\right)$. We then have that

$$
t_{\overline{H O M}_{\tilde{A}[[T]]}(\Pi(Q), \Upsilon(X))} \circ \iota_{*}=\Upsilon\left(t_{X}\right)_{*} \circ \iota_{*}
$$

in $\mathcal{H}\left(\tilde{A}[[T]]^{o p}\right)$. Applying the functor $q: \mathcal{H}\left(\tilde{A}[[T]]^{o p}\right) \longrightarrow \mathcal{D}\left(\tilde{A}[[T]]^{o p}\right)$ to this last equality and bearing in mind that $q\left(\iota_{*}\right)$ is an isomorphism, we conclude that

$$
q\left(\left(\Upsilon\left(t_{X}\right)\right)_{*}\right)=q\left(t_{\overline{H O M}_{\tilde{A}[[T]]}(\Pi(Q), \Upsilon(X))}\right)=t_{\mathbb{R} H_{\tilde{A}}[[T]]}(Q, X) .
$$

For our next result we adopt the terminology of [11, Proposition 9] and, for the given dg algebra with enough idempotents $A$, we put $\mathcal{C}_{V}^{o}=\operatorname{per}(\tilde{A}[[T]])$, we denote by $\mathcal{C}_{V}^{o}\left[t^{-1}\right]$ the localization of $\mathcal{C}_{V}^{o}$ with respect to natural transformation $t$ given above (see [11, Remark 2] for the definition) and we let $p: \mathcal{C}_{V}^{o} \longrightarrow \mathcal{C}_{V}^{o}\left[t^{-1}\right]$ be the canonical functor. We also put ${ }_{V} \mathcal{C}^{o}=\operatorname{per}\left(\tilde{A}[[T]]^{o p}\right),{ }_{V} \mathcal{C}^{o}\left[t^{-1}\right]$ and $p:{ }_{V} \mathcal{C}^{o} \longrightarrow{ }_{V} \mathcal{C}^{o}\left[t^{-1}\right]$ for the corresponding concepts on the left.

Lemma 18. Let $p: \mathcal{C}_{V}^{o} \longrightarrow \mathcal{C}_{V}^{o}\left[t^{-1}\right]$ and $p^{\prime}:{ }_{V} \mathcal{C}^{o} \longrightarrow{ }_{V} \mathcal{C}^{o}\left[t^{-1}\right]$ be the canonical triangulated functors given by localization, and let $Q_{1}$ and $Q_{2}$ be objects of $\mathcal{C}_{V}^{o}=\operatorname{per}(\tilde{A}[[T]])$. There is an isomorphism $p\left(Q_{1}\right) \cong p\left(Q_{2}\right)$ if, and only if, there is an isomorphism $p^{\prime}\left(Q_{1}^{\star}\right) \cong p^{\prime}\left(Q_{2}^{\star}\right)$, where $(?)^{\star}:=\mathbb{R} \operatorname{Hom}_{\tilde{A}[[T]]}(?, \tilde{A}[[T]]): \mathcal{D}(\tilde{A}[[T]])^{o p}=\mathcal{C}_{V}^{o p} \longrightarrow{ }_{V} \mathcal{C}=\mathcal{D}\left(\tilde{A}^{o p}[[T]]\right)$ is the usual triangulated functor.

Proof. The fact that $p\left(Q_{1}\right)$ and $p\left(Q_{2}\right)$ are isomorphic in $\mathcal{C}_{V}^{o}\left[t^{-1}\right]$ means that we have morphisms $f: Q_{1} \longrightarrow Q_{2}$ and $g: Q_{2} \longrightarrow Q_{1}$ in $\mathcal{C}_{V}^{o}=\operatorname{per}(\tilde{A}[[T]])$ such that $g \circ f \circ t_{Q_{1}}^{r}=t_{Q_{1}}^{s}$ and $f \circ g \circ t_{Q_{2}}^{m}=t_{Q_{2}}^{n}$, for some $r, s, m, n \in \mathbb{N}$. If we now apply the duality

$$
(?)^{\star}=\mathbb{R H o m}_{\tilde{A}[[T]]}(?, \tilde{A}[[T]]): \operatorname{per}(\tilde{A}[[T]])=\mathcal{C}_{V}^{o} \stackrel{\cong^{o}}{\longrightarrow} \mathcal{C}^{o}=\operatorname{per}\left(\tilde{A}[[T]]^{o p}\right),
$$

then we get that $\left(t_{Q_{2}}^{\star}\right)^{m} \circ g^{\star} \circ f^{\star}=\left(t_{Q_{2}}^{\star}\right)^{n}$. But Propositions 2 and 17 tell us that we have $\left(t_{Q_{k}}\right)^{\star}=t_{Q_{k}^{\star}}$ for $k=1,2$, which implies that $p^{\prime}\left(g^{\star}\right) \circ p^{\prime}\left(f^{\star}\right)$ and $p^{\prime}\left(f^{\star}\right) \circ p^{\prime}\left(g^{\star}\right)$ are isomorphisms in ${ }_{V} \mathcal{C}^{o}\left[t^{-1}\right]$, and hence that $p^{\prime}\left(Q_{1}^{\star}\right) \cong p^{\prime}\left(Q_{2}^{\star}\right)$ in ${ }_{V} \mathcal{C}^{o}\left[t^{-1}\right]$.

The reverse implication follows by exchanging the roles of $A$ and $A^{o p}$ and of $Q_{k}$ and $Q_{k}^{\star}$, bearing in mind that $Q_{k}$ is isomorphic to $Q_{k}^{\star \star}$, for $k=1,2$ (see [10, Proposition 10.4]). 
The first assertion of the following Lemma 19 seems to be folklore, but we include a short proof. A right $\operatorname{dg} \tilde{A}[[T]]$-module $Q$ will be called T-torsion-free when $t_{Q}: Q \longrightarrow$ $Q$ is monomorphism in $G r-\tilde{A}[[T]]$. Note that this is equivalent to saying that $t_{Q}$ is a monomorphism for the abelian structure of $\mathcal{C}(\tilde{A}[[T]])$.

Lemma 19. Let $A$ be a dg algebra with enough idempotents and let $q_{A}: \mathcal{H}(A) \longrightarrow \mathcal{D}(A)$ be the canonical functor. The following assertions hold:

(1) The induced functor $q: \operatorname{thick}_{\mathcal{H}(A)}\left(e_{i} A: i \in I\right) \longrightarrow \operatorname{per}(A)=\mathcal{D}^{c}(A)$ is an equivalence of triangulated categories.

(2) Each $Q \in \operatorname{thick}_{\mathcal{H}(\tilde{A}[[T]])}\left(e_{i} \tilde{A}[[T]]: i \in I\right)$ is isomorphic in $\mathcal{H}(\tilde{A}[[T]])$ to a T-torsionfree right $d g \tilde{A}[[T]]$-module.

Proof. (1) The subcategory thick $\mathcal{H}_{(A)}\left(e_{i} A: i \in I\right)$ of $\mathcal{H}(A)$ consists of homotopically projective objects and the restriction of $q$ to the subcategory of homotopically projective objects is fully faithful. In order to prove the density, recall that $\operatorname{per}(A)=\operatorname{thick}_{\mathcal{D}(A)}\left(e_{i} A: i \in I\right)$ (see [5, Theorem 5.3]). This implies in particular that each $X \in \operatorname{per}(A)$ is a direct summand in $\mathcal{D}(A)$ of a right dg $A$-module $P$ for which there is a sequence of morphisms

$$
0=P_{0} \stackrel{f_{1}}{\longrightarrow} P_{1} \stackrel{f_{2}}{\longrightarrow} \cdots \stackrel{f_{n-1}}{\longrightarrow} P_{n-1} \stackrel{f_{n}}{\longrightarrow} P_{n}
$$

in $\mathcal{D}(A)$ such that $P_{n}=P$ and $\operatorname{cone}\left(f_{k}\right) \cong e_{i_{k}} A\left[m_{k}\right]$, for some $i_{k} \in I$ and some $m_{k} \in \mathbb{Z}$, for $k=1, \ldots, n$. We will prove by induction on $n$ that $P \cong q(Q)$, for some $Q \in \operatorname{thick}_{\mathcal{H}(A)}\left(e_{i} A: i \in\right.$ $I)$. For $n=0$ there is nothing to prove, so we assume that $n>1$. By the induction hypothesis, we can choose $Q_{n-1} \in \operatorname{thick}_{\mathcal{H}(A)}\left(e_{i} A: i \in I\right)$ such that $q\left(Q_{n-1}\right) \cong P_{n-1}$. We then get a distinguished triangle $Q_{n-1} \longrightarrow P \longrightarrow e_{i} A[m] \stackrel{f[1]}{\longrightarrow} Q_{n-1}[1]$, for some $i \in I$, some $m \in \mathbb{Z}$ and some morphism $f: e_{i} A[m] \longrightarrow Q_{n-1}$ in $\mathcal{D}(A)$. But the functor $q$ gives an isomorphism $\operatorname{Hom}_{\mathcal{H}(A)}\left(e_{i} A, Q_{n-1}\right) \stackrel{\cong}{\longrightarrow} \operatorname{Hom}_{\mathcal{D}(A)}\left(e_{i} A, Q_{n-1}\right)$. This means that we may view $f$ as a morphism in $\mathcal{H}(A)$, and then the triangulated cone $Q=\operatorname{cone}_{\mathcal{H}(A)}(f)$ is in $\operatorname{thick}_{\mathcal{H}(A)}\left(e_{i} A: i \in I\right)$ and satisfies that $q(Q) \cong P$.

Let now $X, P$ and $Q$ be as above and let $e \in \operatorname{End}_{\mathcal{D}(A)}(P)$ be the idempotent endomorphism corresponding to the direct summand $X$ of $P$. Since $q$ gives an algebra isomorphism $\operatorname{End}_{\mathcal{H}(A)}(Q) \stackrel{\cong}{\longrightarrow} \operatorname{End}_{\mathcal{D}(A)}(P)$, we have a unique $\epsilon=\epsilon^{2} \in \operatorname{End}_{\mathcal{H}(A)}(Q)$ such that $q(\epsilon)=e$. Since $\mathcal{H}(A)$ has arbitrary (set-indexed) coproducts, we know that idempotents split in $\mathcal{H}(A)$ (see [ㅁ, Proposition 1.6.8]). We then get a direct summand $Y$ of $Q$ in $\mathcal{H}(A)$ corresponding to $\epsilon$, and we clearly have that $q(Y) \cong X$.

(2) Let $Q \in \operatorname{thick}_{\mathcal{H}(\tilde{A}[[T]])}\left(e_{i} \tilde{A}[[T]]: i \in I\right)$ be any object. By the obvious adaptation of $[5$, Theorem 3.1] to the language of dg algebras with enough idempotents, we know that there is a chain of inflations in $\mathcal{C}(\tilde{A}[[T]])$

$$
0=P_{0} \hookrightarrow P_{1} \hookrightarrow \ldots \hookrightarrow P_{n} \hookrightarrow \ldots
$$

such that $\operatorname{Coker}\left(P_{n-1} \hookrightarrow P_{n}\right)$ is a direct summand in $\mathcal{C}(\tilde{A}[[T]])$ of a (possibly infinite) coproduct of dg right $\tilde{A}[[T]]$-module of the form $e_{i} \tilde{A}[[T]][m]$, with $i \in I$ and $m \in \mathbb{Z}$, and $P=\bigcup_{n \in \mathbb{N}} P_{n}$ is isomorphic to $Q$ in $\mathcal{D}(\tilde{A}[[T]])$. Since all the exact sequences

$$
0 \rightarrow P_{n-1} \hookrightarrow P_{n} \longrightarrow P_{n} / P_{n-1} \rightarrow 0
$$

split in $G r-\tilde{A}[[T]]$, we readily see that $P$ is projective in this category. In particular $P$ is $T$-torsion-free. But $P$ and $Q$ are homotopically projective objects of $\mathcal{H}(\tilde{A}[[T]])$, which implies that the canonical functor $q: \mathcal{H}(\tilde{A}[[T]]) \longrightarrow \mathcal{D}(\tilde{A}[[T]])$ induces bijections $\operatorname{Hom}_{\mathcal{H}(\tilde{A}[[T]])}(X, Y) \stackrel{\cong}{\longrightarrow} \operatorname{Hom}_{\mathcal{D}(\tilde{A}[[T]])}(X, Y)$, for $X, Y \in\{P, Q\}$. We deduce that any isomorphism $P \stackrel{\cong}{\longrightarrow} Q$ in $\mathcal{D}(\tilde{A}[[T]])$ can be lifted to a corresponding isomorphism in $\mathcal{H}(\tilde{A}[[T]])$. 
4.2. The main theorem under hypothesis (b). We can now complete the proof of Theorem 1.

Theorem 20. Let $\mathcal{C}_{k}^{0}$ be the category of compact objects of an algebraic compactly generated triangulated category. For any objects $M, N \in O b\left(\mathcal{C}_{k}^{0}\right)$, the following assertions are equivalent:

(1) There is a distinguished triangle $Z_{\ell} \stackrel{\left(\begin{array}{l}v \\ u\end{array}\right)}{\longrightarrow} Z_{\ell} \oplus M \stackrel{\left(\begin{array}{ll}h & j\end{array}\right)}{\longrightarrow} N \longrightarrow Z_{\ell}[1]$, where $v$ is a nilpotent endomorphism of $Z_{\ell}$.

(2) There is a distinguished triangle $N \stackrel{\left(\begin{array}{l}j \\ h\end{array}\right)}{\longrightarrow} M \oplus Z_{r} \stackrel{\left(\begin{array}{ll}u & v\end{array}\right)}{\longrightarrow} Z_{r} \longrightarrow N[1]$, where $v$ is a nilpotent endomorphism of $Z_{r}$.

Proof. Using the version of Keller's theorem for dg algebras with enough idempotents (see [10, Corollary 6.11]), we can and shall assume that $\mathcal{C}_{k}^{o}=\mathcal{D}(A)^{c}=\operatorname{per}(A)$, where $A$ is a $\mathrm{dg}$ algebra with enough idempotents.

$(1) \Longrightarrow(2)$ : In [11, Proposition 9] we showed that if there is a distinguished triangle as in assertion 1 , then the quintuple $\left(\mathcal{C}_{k}, \mathcal{C}_{V}, \mathcal{C}_{V}^{o}, \uparrow V, t\right)$ give degeneration data for $\mathcal{C}_{k}^{o}$, where $\mathcal{C}_{k}=\mathcal{D}(A), \mathcal{C}_{V}=\mathcal{D}(\tilde{A}[[T]]), \mathcal{C}_{V}^{o}=\mathcal{D}(\tilde{\mathcal{A}}[[\mathcal{T}]])^{c}=\operatorname{per}(\tilde{A}[[T]])$ and $\uparrow_{k}^{V}: \mathcal{D}(A) \longrightarrow \mathcal{D}(\tilde{A}[[T]])$ and $t: 1_{\mathcal{D}(\tilde{A}[[T]])} \longrightarrow 1_{\mathcal{D}(\tilde{A}[[T]])}$ are as in the previous results of this section. Moreover, in the above mentioned result [11, Proposition 9] it was also proved that there exists an object $Q \in \mathcal{C}_{V}^{o}=\operatorname{per}(\tilde{A}[[T]])$ so that both required conditions for categorical degeneration are satisfied, namely:

(1) If $p: \mathcal{C}_{V}^{o} \longrightarrow \mathcal{C}_{V}^{o}\left[t^{-1}\right]$ is the canonical functor, then $p(Q) \cong p\left(M \uparrow_{k}^{V}\right)$;

(2) $\phi\left(\right.$ cone $\left.\left(t_{Q}\right)\right) \cong N$, where $\phi: \mathcal{C}_{V}^{o}=\operatorname{per}(\tilde{A}[[T]]) \longrightarrow \mathcal{D}(A)=\mathcal{C}_{k}$ is the restriction of $\iota_{*}: \mathcal{D}(\tilde{A}[[T]]) \longrightarrow \mathcal{D}(A)$ to $\operatorname{per}(\tilde{A}[[T]])$.

Here and in the rest of the proof cone $(f)$ denotes the triangulated cone. With this information in mind, we give the proof of the theorem, which is divided in two steps:

Step 1: If $Q_{1}$ is a T-torsion-free right $d g \tilde{A}[[T]]$-module in thick ${ }_{\mathcal{H}(\tilde{A}[[T]])}\left(e_{i} \tilde{A}[[T]]: i \in I\right)$ and if we put $Q_{1}^{\star}=\overline{H O M}_{\tilde{A}[[T]]}\left(Q_{1}, \tilde{A}[[T]]\right)$, then

$$
\phi\left(\operatorname{cone}\left(t_{Q_{1}^{\star}}\right)\right) \cong \mathbb{R} \operatorname{Hom}_{A}(?, A)\left(\phi\left(\operatorname{cone}\left(t_{Q_{1}}\right)\right)\right)
$$

Note that $Q_{1}$ is homotopically projective, so that we also have

$$
Q_{1}^{\star}=\mathbb{R H o m}_{\tilde{A}[[T]]}(?, \tilde{A}[[T]])\left(Q_{1}\right) \cong \mathbb{R H O M}_{\tilde{A}[[T]]}\left(Q_{1}, \tilde{A}[[T]]\right)
$$

(see Proposition 2). On the other hand, the homomorphism of dg algebras

$$
\rho \otimes \rho^{o}: \tilde{A}[[T]] \otimes \tilde{A}[[T]]^{o p} \longrightarrow A \otimes A^{o p}
$$

gives a restriction of scalars functor

$$
\left(\rho \otimes \rho^{o}\right)_{*}: A-D g-A \longrightarrow \tilde{A}[[T]]-D g-\tilde{A}[[T]] .
$$

In particular $A$ is a $\operatorname{dg} \tilde{A}[[T]]-\tilde{A}[[T]]$-bimodule by defining $\left(\sum_{k \in \mathbb{N}} a_{k} T^{k}\right) a=a_{0} a$ and $\left.a\left(\sum_{k \in \mathbb{N}} a_{k} T^{k}\right)\right)=a a_{0}$, for all homogeneous elements $\sum_{k \in \mathbb{N}} a_{k} T^{k} \in \tilde{A}[[T]]$ and $a \in A$. Note that we then have an exact sequence of T-symmetric $\tilde{A}[[T]]-\tilde{A}[[T]]$-bimodules

$$
0 \rightarrow \tilde{A}[[T]] \stackrel{t_{\tilde{A}[[T]]}}{\longrightarrow} \tilde{A}[[T]] \stackrel{\rho}{\longrightarrow} A \rightarrow 0
$$

in $G r-\left(\tilde{A}[[T]] \otimes \tilde{A}[[T]]^{o p}\right)$ and in (the abelian structure of $) \mathcal{C}\left(\tilde{A}[[T]] \otimes \tilde{A}[[T]]^{o p}\right)$.

The last sequence gives a distinguished triangle

$$
\tilde{A}[[T]] \stackrel{t_{\tilde{A}[[T]]}}{\longrightarrow} \tilde{A}[[T]] \stackrel{\rho}{\longrightarrow} A \longrightarrow \tilde{A}[[T]][1]
$$


in $\mathcal{D}\left(\tilde{A}[[T]] \otimes \tilde{A}[[T]]^{o p}\right)$. By Propositions 2 and 17 , application of the functor $\mathbb{R H o m}_{\tilde{A}[[T]]}\left(Q_{1}, ?\right)$ : $\mathcal{D}\left(\tilde{A}[[T]] \otimes \tilde{A}[[T]]^{o p}\right) \longrightarrow \mathcal{D}\left(\tilde{A}[[T]]^{o p}\right)$ to the last distinguished triangle gives a distinguished triangle

$$
Q_{1}^{\star} \stackrel{t_{Q_{1}^{\star}}}{\longrightarrow} Q_{1}^{\star} \longrightarrow \mathbb{R H o m}_{\tilde{A}[[T]]}\left(Q_{1}, A\right) \longrightarrow Q_{1}^{\star}[1]
$$

in $\mathcal{D}\left(\tilde{A}[[T]]^{o p}\right)$, so that cone $\left(t_{Q_{1}^{\star}}\right) \cong \mathbb{R H o m}_{\tilde{A}[[T]]}\left(Q_{1}, A\right):=\mathbb{R H o m}_{\tilde{A}[[T]]}\left(Q_{1}, ?\right)(A)$.

It is important to notice that, by Proposition 2 again, we have isomorphisms in $\mathcal{D}\left(\tilde{A}[[T]]^{o p}\right)$ :

$$
\mathbb{R H o m}_{\tilde{A}[[T]]}\left(Q_{1}, ?\right)(A) \cong \mathbb{R H O M}_{\tilde{A}[[T]]}\left(Q_{1}, A\right) \cong \mathbb{R H o m}_{\tilde{A}[[T]]}(?, A)\left(Q_{1}\right)
$$

When we apply the contravariant triangulated functor $\mathbb{R H o m}_{\tilde{A}[[T]]}(?, A): \mathcal{D}(\tilde{A}[[T]]) \longrightarrow$ $\mathcal{D}\left(\tilde{A}[[T]]^{o p}\right)$ to the morphisms $t_{Q_{1}}: Q_{1} \longrightarrow Q_{1}$ we obtain the zero map. Indeed, due to the homotopically projective condition of $Q_{1}$, we have that $\mathbb{R H o m}_{\tilde{A}[[T]]}(?, A)\left(Q_{1}\right)=$ $\overline{H O M}_{\tilde{A}[[T]]}\left(Q_{1}, A\right)$. But since multiplication by $T$ kills the elements of $A$, for each homogeneous element $f \in \overline{H O M}_{\tilde{A}[[T]]}\left(Q_{1}, A\right)$ we have

$$
\left[\mathbb{R H o m}_{\tilde{A}[[T]]}(?, A)\left(t_{Q_{1}}\right)\right](f)=(-1)^{|f|\left|t_{Q_{1}}\right|} f \circ t_{Q_{1}}=f \circ t_{Q_{1}},
$$

and this is a morphism of right $\operatorname{dg} \tilde{A}[[T]]$-modules $Q_{1} \longrightarrow A$ such that

$$
\left(f \circ t_{Q_{1}}\right)(x)=f(x T)=f(x) T=0
$$

for all $x \in Q_{1}$. On the other hand, the contravariant $\operatorname{dg}$ functor $\overline{H O M}_{\tilde{A}[[T]]}(?, A): G r-$ $\tilde{A}[[T]] \longrightarrow \tilde{A}[[T]]-G r$ is left exact and we have an exact sequence

$$
0 \rightarrow Q_{1} \stackrel{t_{Q_{1}}}{\longrightarrow} Q_{1} \stackrel{p}{\longrightarrow} Q_{1} / T Q_{1} \rightarrow 0
$$

in $G r-\tilde{A}[[T]]$ (which is actually an exact sequence in $\mathcal{C}(\tilde{A}[[T]])$ ) due to the T-torsion-free condition of $Q_{1}$. It follows that we have an exact sequence

$$
0 \rightarrow \overline{H O M}_{\tilde{A}[[T]]}\left(Q_{1} / T Q_{1}, A\right) \stackrel{p^{*}}{\longrightarrow} \overline{H O M}_{\tilde{A}[[T]]}\left(Q_{1}, A\right) \stackrel{0}{\longrightarrow} \overline{H O M}_{\tilde{A}[[T]]}\left(Q_{1}, A\right)
$$

in $\tilde{A}[[T]]-G r$. Therefore we have an isomorphism

$$
p^{*}: \overline{H O M}_{\tilde{A}[[T]]}\left(Q_{1} / T Q_{1}, A\right) \stackrel{\cong}{\longrightarrow} \overline{H O M}_{\tilde{A}[[T]]}\left(Q_{1}, A\right) .
$$

We will see that $p^{*}$ commutes with the differentials, which will show that we have an isomorphism

$$
\operatorname{cone}\left(t_{Q_{1}^{\star}}\right) \cong \overline{H O M}_{\tilde{A}[[T]]}\left(Q_{1} / T Q_{1}, A\right)
$$

in $\mathcal{D}\left(\tilde{A}[[T]]^{o p}\right)$. Indeed if $d: \overline{H O M}_{\mathcal{A}[[T]]}\left(Q_{1} / Q_{1} T, A\right) \longrightarrow \overline{H O M}_{\mathcal{A}[[T]]}\left(Q_{1} / Q_{1} T, A\right)$ and $\delta$ : $\overline{H O M}_{\mathcal{A}[[T]]}\left(Q_{1}, A\right) \longrightarrow \overline{H O M}_{\mathcal{A}[[T]]}\left(Q_{1}, A\right)$ denote the respective differentials, then, for each homogeneous element $g \in \overline{H O M}_{\mathcal{A}[[T]]}\left(Q_{1} / Q_{1} T, A\right)$, we have

$$
\left(\delta \circ p^{*}\right)(g)=\delta(g \circ p)=d_{A} \circ g \circ p-(-1)^{|g \circ p|} g \circ p \circ d_{Q_{1}}=d_{A} \circ g \circ p-(-1)^{|g|} g \circ p \circ d_{Q_{1}} \text {. }
$$

But $p$ is a morphism in $Z^{0}(\tilde{A}[[T]]-D g)=\mathcal{C}\left(\tilde{A}[[T]]^{o p}\right)$, so that $d_{Q_{1} / Q_{1} T} \circ p-p \circ d_{Q_{1}}=0$. We then get

$$
\left(\delta \circ p^{*}\right)(g)=d_{A} \circ g \circ p-(-1)^{|g|} g \circ d_{Q_{1} / Q_{1} T} \circ p=d(g) \circ p=\left(p^{*} \circ d\right)(g),
$$

and hence $\delta \circ p^{*}=p^{*} \circ d$ as desired.

If we apply the restriction of scalars functor $\phi=\iota_{*}: \tilde{A}[[T]]-D g \longrightarrow A-D g$, then $\overline{H O M}_{\tilde{A}[[T]]}\left(Q_{1} / T Q_{1}, A\right)$ is taken to $\overline{H O M}_{A}\left(\phi\left(Q_{1} / Q_{1} T\right), A\right)$. But, as right $\operatorname{dg} A$-modules, we have an isomorphism $\phi\left(Q_{1} / Q_{1} T\right)=\rho^{*}\left(Q_{1}\right)$ where $\rho^{*}=? \otimes_{\tilde{A}[[T]]} A: D g-\tilde{A}[[T]] \longrightarrow D g-A$ is the extension of scalars along the morphism of dg algebras with enough idempotents $\rho: \tilde{A}[[T]] \longrightarrow A$. Then the induced triangulated functor

$$
\rho^{*}=H^{0}\left(\rho^{*}\right): H^{0}(D g-\tilde{A}[[T]])=\mathcal{H}(\tilde{A}[[T]]) \longrightarrow \mathcal{H}(A)=H^{0}(D g-A)
$$


has the property that

$$
\rho^{*}\left(Q_{1}\right)=\phi\left(Q_{1} / Q_{1} T\right) \in \operatorname{thick}_{\mathcal{H}(A)}\left(\rho^{*}\left(e_{i} \tilde{A}[[T]]\right): i \in I\right)=\operatorname{thick}_{\mathcal{H}(A)}\left(e_{i} A: i \in I\right) .
$$

In particular, we get that $\phi\left(Q_{1} / Q_{1} T\right)$ is homotopically projective in $\mathcal{H}(A)$ and, hence, that

$$
\overline{H O M}_{A}\left(\phi\left(Q_{1} / Q_{1} T\right), A\right) \cong \mathbb{R} \operatorname{Hom}_{A}(?, A)\left(\phi\left(Q_{1} / Q_{1} T\right)\right) \cong \mathbb{R} \operatorname{HOM}_{A}\left(\phi\left(Q_{1} / Q_{1} T\right), A\right) .
$$

(see Proposition 2). Bearing in mind that the exact sequence

$$
0 \rightarrow Q_{1} \stackrel{t_{Q_{1}}}{\longrightarrow} Q_{1} \longrightarrow Q_{1} / T Q_{1} \rightarrow 0
$$

in $\mathcal{C}(\tilde{A}[[T]])$ (with respect to the abelian exact structure of $\mathcal{C}(\tilde{A}[[T]]))$ gives a distinguished triangle

$$
Q_{1} \stackrel{t_{Q_{1}}}{\longrightarrow} Q_{1} \longrightarrow Q_{1} / T Q_{1} \longrightarrow Q_{1}[1]
$$

in $\mathcal{D}(\tilde{A}[[T]])$, we get that $Q_{1} / Q_{1} T \cong \operatorname{cone}\left(t_{Q_{1}}\right)$ in $\mathcal{D}(\tilde{A}[[T]])$. We then get isomorphisms

$$
\begin{aligned}
\phi\left(\operatorname{cone}\left(t_{Q_{1}^{\star}}\right)\right) & \cong \phi\left(\overline{H O M}_{\tilde{A}[[T]]}\left(Q_{1} / T Q_{1}, A\right)\right) \\
& \cong \overline{H O M}_{A}\left(\phi\left(Q_{1} / Q_{1} T\right), A\right) \\
& \cong \mathbb{R H o m}_{A}(?, A)\left(\phi\left(\operatorname{cone}\left(t_{Q_{1}}\right)\right)\right) .
\end{aligned}
$$

Step 2: End of the proof: Let now $M$ and $N$ be as in assertion (1) and let $Q \in \mathcal{C}_{V}^{o}=$ $\operatorname{per}(\tilde{A}[[T]])$ be the right $\operatorname{dg} \tilde{A}[[T]]$-module considered in the second paragraph of this proof. Using Lemma 19, without loss of generality, we may assume that $M, N \in \operatorname{thick}_{\mathcal{H}(A)}\left(e_{i} A: i \in\right.$ $I)$ and that $Q$ is a T-torsion-free right $\tilde{A}[[T]]$-module in $\operatorname{thick}_{\mathcal{H}(\tilde{A}[[T]])}\left(e_{i} \tilde{A}[[T]]: i \in I\right)$. Note that then $\tilde{M}[[T]] \in \operatorname{thick}_{\mathcal{H}(\tilde{A}[[T]])}\left(e_{i} \tilde{A}[[T]]: i \in I\right)$ and $\tilde{M}[[T]]$ is T-torsion-free.

According to the Step 1, we have that

$$
\phi\left(\operatorname{cone}\left(t_{Q^{*}}\right)\right) \cong \mathbb{R} \operatorname{Hom}_{A}(?, A)\left(\phi\left(\operatorname{cone}\left(t_{Q}\right)\right)\right)=\mathbb{R} \operatorname{Hom}_{A}(?, A)(N)=: N^{*} .
$$

On the other hand, by Corollary 15 and Proposition 14, we know that

$$
\left(M \uparrow_{k}^{V}\right)^{\star}:=\mathbb{R H o m}_{\tilde{A}[[T]]}(?, \tilde{A}[[T]])\left(M \uparrow_{k}^{V}\right) \cong M^{*} \uparrow_{k}^{V},
$$

where $M^{*}:=\mathbb{R H o m}_{A}(?, A)(M)$. Moreover, by Lemma 18, we get that $p^{\prime}\left(\left(M \uparrow_{k}^{V}\right)^{\star}\right)$ and $p^{\prime}\left(Q^{\star}\right)$ are isomorphic in ${ }_{V} \mathcal{C}^{o}\left[t^{-1}\right]$, where $p^{\prime}:{ }_{V} \mathcal{C}^{o} \longrightarrow{ }_{V} \mathcal{C}^{o}\left[t^{-1}\right]$ is the canonical functor. It follows from this that the left $\operatorname{dg} \tilde{A}[[T]]$-module

$$
Q^{\star}:=\mathbb{R H o m}_{\tilde{A}[[T]]}(?, \tilde{A}[[T]])(Q)=\mathbb{R H O M}_{\tilde{A}[[T]]}(Q, \tilde{A}[[T]])
$$

defines a categorical degeneration $M^{*} \leq_{c d e g} N^{*}$, where $(?)^{*}=\mathbb{R H o m}_{A}(?, A): \operatorname{per}(A) \stackrel{\cong}{\stackrel{0}{\longrightarrow}}$ $\operatorname{per}\left(A^{o p}\right)$ is the duality defined by the regular $\operatorname{dg}$ bimodule $X=A$ (see [10, Proposition 10.4]).

But categorical degeneration implies triangle degeneration by Theorem 7 (see [11, Proposition 8]), so that we have $M^{*} \leq_{\Delta+\text { nil }} N^{*}$. That is, we have a distinguished triangle

$$
U \stackrel{\left(\begin{array}{l}
v^{\prime} \\
u^{\prime}
\end{array}\right)}{\longrightarrow} U \oplus M^{*} \stackrel{\left(\begin{array}{ll}
h^{\prime} & j^{\prime}
\end{array}\right)}{\longrightarrow} N^{*} \longrightarrow U[1]
$$

in ${ }_{k} \mathcal{C}^{o}=\operatorname{per}\left(A^{o p}\right)$, where $v^{\prime}$ is a nilpotent endomorphism of $U$. Applying the duality $\mathbb{R H o m}_{A^{o p}}(?, A): \operatorname{per}\left(A^{o p}\right) \stackrel{\cong}{\longrightarrow} \operatorname{per}(A)$ to this last distinguished triangle, we obtain a distinguished triangle

$$
N \stackrel{\left(\begin{array}{l}
j^{\prime} * \\
h^{\prime *}
\end{array}\right)}{\longrightarrow} M \oplus U^{*} \stackrel{\left(\begin{array}{ll}
u^{*} & v^{\prime *}
\end{array}\right)}{\longrightarrow} U^{*} \longrightarrow N[1],
$$


where $v^{\prime *}$ is clearly a nilpotent endomorphism of $U^{*}$. The proof of the implication ends by taking $Z_{r}:=U^{*}$.

$(2) \Longrightarrow(1):$ By applying the duality $\mathbb{R H o m}_{A}(?, A): \mathcal{C}_{k}^{o}=\operatorname{per}(A) \stackrel{\cong o}{\longrightarrow} \operatorname{per}\left(A^{o p}\right)$ to the distinguished triangle in assertion (2) we get that $M^{*} \leq \Delta+$ nil $N^{*}$ in ${ }_{k} \mathcal{C}^{o}:=\operatorname{per}\left(A^{o p}\right)$. The proof of the implication 1$) \Longrightarrow 2$ ), when applied with $A^{o p}$ instead of $A$, shows that we have $M \cong M^{* *} \leq_{\Delta+\text { nil }} N^{* *} \cong N$, so that the distinguished triangle of assertion 1) exists.

Observe that the proof actually gives the following additional statement.

Corollary 21. Let $\mathcal{C}_{k}^{0}=\mathcal{D}(A)^{c}$ be the category of compact objects in the derived category $\mathcal{D}(A)$ of a dg algebra $A$ with enough idempotents. For any objects $M, N \in O b\left(\mathcal{C}_{k}^{0}\right)$, we get

$$
M \leq_{\Delta+\text { nil }} N \Leftrightarrow \mathbb{R} \operatorname{Hom}_{A}(?, A)(M) \leq_{\Delta+\text { nil }} \mathbb{R} \operatorname{Hom}_{A}(?, A)(N) .
$$

\section{REFERENCES}

[1] Theo Bühler, Exact categories, Expositiones Mathematicae 28(1) (2010) 1-69

[2] Xiao-Wu Chen, Yu Ye and Pu Zhang, Algebras of derived dimension zero, Communications in Algebra 36 (2008) 1-10.

[3] Dieter Happel, Triangulated Categories in the Representation Theory of Finite Dimensional Algebras, London Mathematical Society Lecture Note Series 119. Cambridge University Press 1988.

[4] Bernt Tore Jensen, Xiuping Su and Alexander Zimmermann, Degeneration-like orders for triangulated categories, Journal of Algebra and Applications 4 (2005) 587-597.

[5] Bernhard Keller, Deriving DG categories, Annales Scientifiques de l'École Normale Supérieure 27 (1994) 63-102.

[6] Bernhard Keller, On differential graded categories, In: International Congress of Mathematics, vol. II. European Mathematical Society Zurich (2006) 151-190.

[7] Bernhard Keller and Sarah Scherotzke, Graded quiver varieties and derived categories, Journal für die reine und angewandte Mathematik 713 (2016) 85-127.

[8] Amnon Neeman, Triangulated Categories, Princeton University Press 2001.

[9] Christine Riedtmann, Degenerations for representations of quivers with relations, Annales Scientifiques de l'École Normale Supérieure 19 (1986) 275-301.

[10] Manuel Saorín, Dg algebras with enough idempotents, their dg modules and their derived categories, Preprint available at https://arxiv.org/abs/1612.04719 (2016).

[11] Manuel Saorín and Alexander Zimmermann, An Axiomatic Approach for Degenerations in Triangulated Categories, Applied Categorical Structures 24 (2016) no 4, 385-405.

[12] Bo Stenström, Rings of QUOTIENTs, Springer-Verlag 1975.

[13] Jean-Louis Verdier, Des catégories dérivées des catégories abeliennes. Astérisque 239, Société Mathématique de France (1996).

[14] Yuji Yohino, On degeneration of modules, Journal of Algebra 278 (2004) 217-226.

[15] Yuji Yoshino, Stable degeneration of Cohen-Macaulay modules, Journal of Algebra 332 (2011) 500-521.

[16] Zhengfang Wang, Triangle order $\leq_{\Delta}$ in Singular Categories, Algebras and Representation Theory 19 (2016) 397-404.

[17] Peter Webb, Consequences of the existence of Auslander-Reiten triangles with applications to perfect complexes for self-injective algebras, preprint 2014;

[18] Alexander Zimmermann, Representation Theory; A homological algebra point of View, Springer Verlag, Cham 2014.

[19] Grzegorz Zwara, A degeneration-like order for modules, Archiv der Mathematik 71 (1998) 437-444.

[20] Grzegorz Zwara, Degenerations of finite dimensional modules are given by extensions, Compositio Mathematica 121 (2000) 205-218. 
Departemento de Matemáticas,

Universidad de Murcia, Aptdo. 4021

30100 Espinardo, Murcia,

SPAIN

E-mail address: msaorinc@um.es

Université DE PiCARDIE,

DÉpartement de Mathématiques et LAMFA (UMR 7352 du CNRS),

33 RUE ST LEU,

F-80039 Amiens Cedex 1,

FRANCE

E-mail address: alexander.zimmermann@u-picardie.fr 